\title{
Maternal-fetal Stress and Dna Methylation Signatures in Neonatal Saliva: an Epigenome-wide Association Study.
}

\section{Ritika Sharma}

Research Unit of Molecular Epidemiology, Institute of Epidemiology, Helmholtz Zentrum Munich, Munich, Germany; Department of Obstetrics and Gynecology, Klinikum rechts der Isar, Technical University of Munich, Germany

\section{Martin G. Frasch}

Department of Obstetrics and Gynecology and Center on Human Development and Disability (CHDD), University of Washington, Seattle, WA, USA

\section{Camila Zelgert}

Department of Obstetrics and Gynecology, Klinikum rechts der Isar, Technical University of Munich, Germany

\section{Peter Zimmermann}

Department of Obstetrics and Gynecology, Klinikum rechts der Isar, Technical University of Munich, Germany

\section{Bibiana Fabre}

Instituto de Fisiopatología y Bioquímica Clínica (INFIBIOC), Facultad de Farmacia y Bioquímica, Universidad de Buenos Aires, Buenos Aires, Argentina

\section{Rory Wilson}

Research Unit of Molecular Epidemiology, Institute of Epidemiology, Helmholtz Zentrum Munich, Munich, Germany

\section{Melanie Waldenberger}

Research Unit of Molecular Epidemiology, Institute of Epidemiology, Helmholtz Zentrum Munich, Munich, Germany

\section{James W. MacDonald}

Department of Environmental and Occupational Health Sciences, University of Washington, Seattle, WA, USA

\section{Theo K. Bammler}

Department of Environmental and Occupational Health Sciences, University of Washington, Seattle, WA, USA

\section{Silvia M. Lobmaier}

Department of Obstetrics and Gynecology, Klinikum rechts der Isar, Technical University of Munich, Germany 
Marta C. Antonelli ( $\square$ mca@fmed.uba.ar)

Department of Obstetrics and Gynecology, Klinikum rechts der Isar, Technical University of Munich, Germany;

\section{Research Article}

Keywords: Pregnancy, Prenatal stress, Perceived stress, Biomarkers, Newborn saliva, DNA methylation, Cortisol, Epigenetics, EWAS

Posted Date: February 11th, 2022

DOI: https://doi.org/10.21203/rs.3.rs-1348030/v1

License: (c) (i) This work is licensed under a Creative Commons Attribution 4.0 International License. Read Full License 


\section{Abstract \\ Background}

Maternal stress before, during and after pregnancy has profound effects on the development and lifelong function of the infant's neurocognitive development. We hypothesized that the programming of the Central Nervous System (CNS), Hypothalamic Pituitary Axis (HPA) and Autonomic Nervous System (ANS) induced by prenatal stress (PS) is reflected in electrophysiological and epigenetic biomarkers. In this study, we aimed to find non-invasive epigenetic biomarkers of PS in the newborn salivary DNA.

\section{Results}

738 pregnant women were screened for stress exposure using Cohen Perceived Stress Scale (PSS), 164 women were enrolled and 114 dyads were analyzed. Prenatal Distress Questionnaire (PDQ) was also administered to assess specific pregnancy worries. Transabdominal fetal Electrocardiograms (taECG) were recorded to derive coupling between maternal and fetal heart rates resulting in a "Fetal Stress Index" (FSI). Upon delivery, we collected maternal hair strands for cortisol measurements and newborn's saliva for epigenetic analyses. DNA was extracted from saliva samples and DNA methylation was measured using EPIC Bead-Chip array (850k CpG sites). Linear regression was used to identify associations between PSS/PDQ/FSI/Cortisol and DNA methylation. We found epigenome-wide significant associations for $5 \mathrm{CpG}$ with PDQ and cortisol at FDR $<5 \%$. Three $\mathrm{CpGs}$ were annotated to genes (Illumina Gene annotation file): YAP1, TOMM20 and CSMD1 and two CpGs were located approximately lay at 50kb from SSBP4 and SCAMP1. In addition, two Differentiated Methylation regions (DMR) related to maternal stress measures PDQ and cortisol were found: DAXX and ARL4D.

\section{Conclusions}

Genes annotated to these $\mathrm{CpGs}$ were found to be involved in secretion and transportation, nuclear signaling, Hippo signaling pathways, apoptosis, intra-cellular trafficking and neuronal signaling. Moreover, some CpGs are annotated to genes related to autism, Post Traumatic Stress Disorder (PTSD) and schizophrenia. Early assessment of such non-invasive PS biomarkers will allow timelier detection of babies at risk and a more effective allocation of resources for early intervention programs to improve child development. A biomarker-guided early intervention strategy is the first step in the prevention of future health problems, reducing their personal and societal impact.

\section{Background}

Compelling evidence both from animal and human studies indicates that adversities in the perinatal environment significantly increase the risk for developing neurocognitive disorders later in life $(1-10)$. 
Human studies provide substantial evidence that maternal stress during the gestational period (namely PS) can lead to behavioral, cognitive and temperamental disorders in the infant, increasing child morbidity and neurological dysfunction. For example, maternal psychosocial stress (general and specific stress and anxiety) increases the risk for the growing infant to develop disorders such as attention-deficit hyperactivity disorder (ADHD) (10), autism spectrum disorders (ASD) (11) and sleep disturbance that can result in depression and other psychiatric disorders. Severe PS is associated with increased cortisol response after a behavioral challenge paradigm in young adults (12). Moreover, several studies have indicated that PS and glucocorticoid exposure may re-program the cardiovascular system, including aberrations in cardiac and kidney development(13).

It is important to understand the mediators that connect the mother' stress with the fetus. In this regard, Rakers et al (8) proposed that the causal pathway lies not only via cortisol but also includes catecholamines, reactive oxygen species, cytokines, serotonin/tryptophan and maternal microbiota. The stress response system has been traditionally linked to the Hypothalamic-Pituitary adrenal (HPA) axis that is responsible for the production and secretion of corticosteroids under basal and stressed conditions (14). However, the Autonomic Nervous system (ANS) also plays a key role through its rapid (within seconds) activation enabling fine adjustments of the target organs. This aspect has been often neglected. In this regard, Monk et al, (15) have shown that the fetal ANS is very perceptive of maternal anxiety. This study shows that during women's recovery following a stress-eliciting task, fetal heart rate (fHR, a biomarker of ANS) changed in association with the mother's acute cardiovascular activity (15). In addition, fHR sensitivity to a stimulus reflects emerging individual differences in the development of ANS $(16,17)$.

Responding to environmental factors, these stress-mediating pathways are assumed to leave permanent epigenetic signatures that may affect the neurobehavioral outcomes of the child. In fact, it has been shown that the epigenome is vulnerable to external exposures during the early prenatal development, a crucial period when intense programming of gene expression is taking place $(18,19)$. Among these mechanisms, DNA methylation is recognized as the most well-characterized epigenetic signature.

While some knowledge has been gained linking the scale of epigenetic organization of phenotypical information with the psychological behavior $(20,21)$ the connection between the epigenetic signatures of adversity and the scale of biophysical organization of human integrative physiology has remained unexplored. This is of great interest because biophysical behavior, such as the properties of ANS describable non-invasively by mathematical HRV analyses, is highly accessible, now more than ever, with the rise of the wearable technologies and remote health monitoring (22). As such, linking these scales of physiological organization may aid in early detection of unhealthy developmental trajectories and timely intervention while also providing a more mechanistic multi-scale framework for understanding these complex relationships (7). With the present "FELICITy" study, we aimed to address this knowledge gap.

First, we hypothesized that early epigenetic signatures of PS are detectable in neonatal saliva. This is important because the detection of such epigenetic signatures, ideally at birth, will help to detect "at-risk 
children" who can benefit from early stimulation programs and follow-up (23). Second, we hypothesized that late gestation biophysical alterations in mother-fetus dyads due to PS are reflected in the neonatal epigenetic marks observed at birth. To test these hypotheses, we devised the FELICITy study to obtain a combination of non-invasive multimodal physiological measures of PS.

We recruited a cohort of third-trimester pregnant women screened for exposure to chronic psychosocial stress during pregnancy. The biophysical signature of the PS exposure has been validated during late gestation using non-invasive feto-maternal transabdominal electrocardiogram (taECG). This approach yielded fetal stress index (FSI), a joint maternal-fetal biomarker of PS (24). At the same time, we also acquired quantitative psychological maternal chronic stress scores (details in Results and Methods)(24). Using maternal behavioral data and the biophysical FSI assessment on one hand and the epigenetic analysis of the newborn's saliva on the other hand, we aimed to validate the presence of linkages between the mother-fetus behavior and the neonatal saliva's epigenetic modifications. To test for persistence of such multi-modal multi-scale linkages we are currently following up this cohort for a second time point at two years of age. Here, we report the existence of such anticipated linkages at birth. The study represents a first report of a well-controlled, prospective study to investigate epigenome-wide methylation changes in $>850.000$ loci in newborn saliva samples in association with behavioral and biophysical maternal-fetal stress measures.

\section{Results}

We performed an Epigenome-Wide Association Study (EWAS) with the Illumina MethylationEPIC BeadChip array, which includes around 850,000-methylation loci in saliva samples obtained from newborns at the time of delivery. We examined the association between prenatal exposure to maternal psychosocial stress and offspring genome-wide saliva methylation using four statistical approaches in association with four complementary maternal and fetal stress measures. The stress measures are: 1) the PSS-10 questionnaire, accounting for the perceived stress of a mother during the third trimester; 2) the PDQ questionnaire accounting for the specific worries related to pregnancy such as pain during labor and delivery, personal appearance after delivery and baby's health; 3) maternal hair cortisol levels (integrated cortisol levels in hair reflecting three prior months of stress exposure) accounting for the chronic activity of the HPA stress response system of the mother during the third trimester and 4) the FSI, a biophysical ANS biomarker for stress, which accounts for fetal ANS reactivity to maternal heart beat during pregnancy.

\section{Cohort characteristics}

The socio-demographic characteristics of the participating mothers and their offspring $(n=114)$ are shown in Table 1. The average age of the mother at study entry was 34 years. Most of the mothers in this study were Caucasians, married and having a university degree. Pregnancies were mostly planned and $72 \%$ of mothers had vaginal deliveries. The perceived stress measures (PSS and PDQ scores) were moderately correlated (Spearman R2 $=0.537$; $\mathrm{p}$-value $=6.952 \mathrm{e}-10$ ). 
Table 1

Baseline characteristics of study population ( $n=114$, mother-newborn pairs), FELICITy study.

\section{Characteristics}

Continuous variables

Age of mother at study entry, years

Birthweight, grams

Gestational age at screening, weeks

Gestational age at inclusion, weeks

Gestational age at birth, weeks

$\mathrm{BMl}$ at study entry, $\mathrm{kg} / \mathrm{m}^{2}$

BMI pregestational, $\mathrm{kg} / \mathrm{m}^{2}$

Score PSS

Score PDQ

Cortisol in maternal hair, $\mathrm{pg} / \mathrm{mg}$

FSI

Categorical variables

Gender, female

European/Caucasian, Others

Married, yes

University degree, yes

Household income $>5000 € /$ month, Yes

Working status at screening, working

Multiparity, Yes

Planned pregnancy, Yes

Cesarean delivery, Yes

Smoking, Yes

IVF / ICSI, Yes

Gestational diabetes, Yes

Autoimmune disease, Yes

\section{$n=114$}

[mean (SD)], [median(range)]

$34.61(4.52)$

$3544.96(431.78)$

$34.07[33.18,34.96]$

$36.71[35.32,37.54]$

39.71 [38.86, 40.57]

27.61 [25.20, 30.42]

$21.82[20.30,24.96]$

$17.00[9.00,22.00]$

$10.50[6.25,16.75]$

$97.00[58.00,161.00]$

$0.15[-0.28,0.60]$

$n(\%)$

52 (45.6)

8 (7.01)

85 (74.6)

80 (70.2)

53 (46.5)

4 (3.5)

85 (74.6)

$92(81.4)$

28 (24.6)

7 (6.1)

$10(8.8)$

11 (9.6)

15 (13.2) 
Displayed are the population characteristics of the FELICITy study population. Continuous variables are given in mean (SD) and median (range) while categorical variables are shown in proportion $\mathrm{n}(\%)$.

Data are mean (SD) using Chi square test, mean (interquartile) using Wilcox. Test or $\mathrm{n}(\%)$ using Fischer's test.

PSS: Cohen perceived stress scale, PDQ: Prenatal distress questionnaire, BMI: Body-mass index; ICSI: Intracytoplasmic sperm injection; IVF: In-vitro-fertilization

\section{Differentially methylated positions (DMPs)}

Each CpG site was separately tested for association with exposure to stress (PSS, PDQ, Cortisol and FSI) and separate linear regression models were run, unless otherwise specified. All the models were adjusted as specified in the methods section.

DNA methylation and Stress measures.

\section{DNA Methylation and PSS score}

PSS score (the continuous variable) was used for the association analysis. We did not identify any differentially methylated sites in relation to PSS score. Figure 1 shows the Manhattan plots. Q-Q plots are shown the supplementary file S1, while Table 2 shows the top four hits for the association with no significant findings.

\section{DNA methylation and PDQ score}

The association analysis with PDQ scores yielded one CpG (cg06542869, p = 4.62E-08) (Fig. 2) achieving FDR $<0.05$. This site has a positive direction of effect and it is located in the body of the protein coding gene YAP1 (Yes1 regulated transcription factor) present in chromosome 11 (Table 2). The regression coefficients and values for the next three non-significant hits for this association are reported in Table 2.

\section{DNA methylation and Cortisol}

We identified stronger associations with cortisol compared to other stress variables (Fig. 3, Table 2). Four CpG sites were identified after controlling for multiple testing using FDR $<0.05$ (cg11409463, cg20905655, cg25252839 and cg05306225). The top hit was cg11409463, located on chromosome 5 but did not annotate to any gene via the Illumina gene annotation file. A look up on University of California Santa Cruz (UCSC) browser showed that the nearest genes within 50kb distance to this CpG site are SCAMP1 (Secretory Carrier Membrane Protein 1) and AP3B1 (Adaptor Related Protein Complex 3 Subunit Beta 1). This CpG site also overlaps with several transcription factors from the AP-1 family. The second hit was cg20905655 ( $p=1.16 \mathrm{E}-07)$ located on chromosome 19 which did not annotate to any gene via Illumina platform. According to the UCSC genome browser, the nearest genes within the $50 \mathrm{~kb}$ region are SSBP4 (Single Stranded DNA Binding Protein 4) (Castro et al., 2002) and LRRC25 (Leucine 
Rich Repeat Containing 25). The third hit was TOMM20 (Translocase of Outer Mitochondrial Membrane 20) (cg25252839, $\mathrm{p}=1.24 \mathrm{E}-07$ ) located on chromosome 1. All the $\mathrm{CpG}$ sites had a positive direction of association except for the fourth hit, cg05306225, which annotates for the gene CSMD1 (CUB And Sushi Multiple Domains 1) located on chromosome 8 and encoding for Q96PZ7-CSMD1_HUMAN (CUB and sushi domain-containing protein 1). Inspection of quantile-quantile (QQ) plot did not show evidence for inflation or bias (Supplementary section S3; lambda $=1.08$ ).

\section{DNA methylation and FSI}

There were no DMPs that survived the correction for multiple testing when the association was performed with FSI, the biophysical biomarker of PS exposure. Of interest, the top hit, CpG (cg13547817, p = 8.51E08) with an FDR: 0.06 , very close to the threshold, mapped to the gene ERP44 (Endoplasmic reticulum protein 44) on chromosome 9 , which is a protein coding gene whose related pathways are the Innate immune system and translational control. Table 2 shows the top four hits from the FSI association analysis and Fig. 4 shows the Manhattan plot. 
Table 2

CpG sites associated with stress measures in DNA methylation analysis.

\begin{tabular}{|c|c|c|c|c|c|c|c|}
\hline $\begin{array}{l}\text { Stress } \\
\text { measure }\end{array}$ & Probe & Coef $^{d}$ & P.Value ${ }^{e}$ & FDR $^{f}$ & chr $r^{9}$ & $\begin{array}{l}\text { Illumina } \\
\text { Gene } \\
\text { annotation }\end{array}$ & $\begin{array}{l}\text { Genes within } \\
50 \mathrm{~kb} \text { of } \\
\text { associated CpG }\end{array}$ \\
\hline \multirow[t]{4}{*}{$\mathrm{PSS}^{\mathrm{a}}$} & $\operatorname{cg} 17478679$ & -0.2 & $\begin{array}{l}3.59 \mathrm{E}- \\
07\end{array}$ & 0.25 & chr17 & KPNB1 & CRHR1 \\
\hline & $\operatorname{cg} 22124215$ & -0.1 & $\begin{array}{l}1.07 \mathrm{E}- \\
06\end{array}$ & 0.25 & Chr2 & MARCH4 & DIRC3 \\
\hline & cg06195987 & 0.52 & $\begin{array}{l}1.96 \mathrm{E}- \\
06\end{array}$ & 0.25 & Chr7 & NA & LMTK2 \\
\hline & $\operatorname{cg} 15426815$ & 0.31 & $\begin{array}{l}2.24 \mathrm{E}- \\
06\end{array}$ & 0.25 & Chr12 & MIR200C & C1S \\
\hline \multirow[t]{4}{*}{$P D Q^{b}$} & $\operatorname{cg} 06542869$ & 0.02 & $\begin{array}{l}4.62 \mathrm{E}- \\
08\end{array}$ & 0.03 & chr11 & YAP1 & YAP1 \\
\hline & $\operatorname{cg} 22861369$ & 0.02 & $\begin{array}{l}2.30 \mathrm{E}- \\
07\end{array}$ & 0.08 & chr5 & PDLIM4 & SLC22A4 \\
\hline & cg01629131 & 0.03 & $\begin{array}{l}3.31 \mathrm{E}- \\
07\end{array}$ & 0.08 & chr20 & NA & RP11, RP1 \\
\hline & cg03105159 & 0.01 & $\begin{array}{l}7.18 \mathrm{E}- \\
07\end{array}$ & 0.11 & chr2 & ALKAL2 & $\begin{array}{l}\text { ALKAL2, } \\
\text { FAM105B }\end{array}$ \\
\hline \multirow[t]{4}{*}{ Cortisol } & $\operatorname{cg} 11409463$ & 0.003 & $\begin{array}{l}2.87 \mathrm{E}- \\
09\end{array}$ & 0.002 & chr5 & NA & SCAMP1 \\
\hline & $\operatorname{cg} 20905655$ & 0.004 & $\begin{array}{l}1.16 \mathrm{E}- \\
07\end{array}$ & 0.03 & chr19 & NA & SSBP4; \\
\hline & $\operatorname{cg} 25252839$ & 0.002 & $\begin{array}{l}1.24 \mathrm{E}- \\
07\end{array}$ & 0.03 & chr1 & TOMM20 & SNORA14B \\
\hline & $\operatorname{cg} 05306225$ & -0.002 & $\begin{array}{l}2.08 \mathrm{E}- \\
07\end{array}$ & 0.04 & chr8 & CSMD1 & CSMD1 \\
\hline $\mathrm{FSI}^{\mathrm{C}}$ & $\operatorname{cg} 13547817$ & -0.39 & $\begin{array}{l}8.85 \mathrm{E}- \\
08\end{array}$ & 0.07 & chr9 & ERP44 & ERP44; INVS \\
\hline
\end{tabular}

The table shows top four $\mathrm{CpGs}$ from the EWAS that are associated to the respective stress measures. Marked in bold are significant.

${ }^{a}$ Cohen perceived stress scale

${ }^{\text {b}}$ Prenatal distress questionnaire

${ }^{\mathrm{C}}$ Fetal stress Index

${ }^{\mathrm{d}}$ Regression coefficients from the statistical model 


\begin{tabular}{|llllllll|}
\hline $\begin{array}{l}\text { Stress } \\
\text { measure }\end{array}$ & Probe & Coef $^{\text {d }}$ & P.Value & FDR $^{\text {f }}$ & chr $^{9}$ & $\begin{array}{l}\text { Illumina } \\
\text { Gene } \\
\text { annotation }\end{array}$ & $\begin{array}{l}\text { Genes within } \\
\text { 50kb of } \\
\text { associated CpG }\end{array}$ \\
\hline cg07642729 & -0.35 & $\begin{array}{l}2.81 \mathrm{E}- \\
06\end{array}$ & 0.49 & chr8 & ASB15 & - \\
\hline cg24795351 & -0.34 & $\begin{array}{l}3.79 \mathrm{E}- \\
06\end{array}$ & 0.49 & chr8 & PREX2 & PREX2 \\
\hline cg16692227 & -0.21 & $\begin{array}{l}3.80 \mathrm{E}- \\
06\end{array}$ & 0.49 & chr14 & SAMD12 & SAMD12 \\
\hline
\end{tabular}

The table shows top four $\mathrm{CpGs}$ from the EWAS that are associated to the respective stress measures. Marked in bold are significant.

${ }^{a}$ Cohen perceived stress scale

${ }^{b}$ Prenatal distress questionnaire

${ }^{\mathrm{c}}$ Fetal stress Index

${ }^{\mathrm{d}}$ Regression coefficients from the statistical model

eSignificance from the statistical model

${ }^{f}$ False discovery rate

${ }^{\text {gChromosome }}$

NA: Not available.

\section{Sex-specificity analysis}

The CpG-by-sex interaction analysis did not reveal any significant differences between sexes for the associations (Table 3). 
Table 3

Epigenome-wide results of the Interaction analysis between Gender and Stress measures.

\begin{tabular}{|c|c|c|c|c|c|c|}
\hline Stress measures & Probe & Coef $^{d}$ & P.Value ${ }^{e}$ & $F^{\prime} R^{f}$ & $\mathrm{Chr}^{\mathrm{g}}$ & Illumina gene annotation \\
\hline \multirow[t]{2}{*}{$\mathrm{PSS}^{\mathrm{a}}$} & $\operatorname{cg} 09723184$ & 0.03 & $6.23 \mathrm{E}-06$ & 0.87 & chr8 & FBX043 \\
\hline & cg27293447 & -0.04 & 7.25E-06 & 0.87 & chr2 & LOC102800447 \\
\hline \multirow[t]{2}{*}{$\mathrm{PDQ}^{\mathrm{b}}$} & $\operatorname{cg} 03756940$ & -0.05 & $1.10 \mathrm{E}-07$ & 0.08 & chr2 & NA \\
\hline & $\operatorname{cg} 00008621$ & -0.04 & 2.06E-07 & 0.08 & chr14 & HIF1A \\
\hline \multirow[t]{2}{*}{ Cortisol } & cg18197866 & 0.003 & 2.55E-07 & 0.16 & $\operatorname{chr} 12$ & PXN \\
\hline & cg20460797 & -0.006 & 4.17E-07 & 0.16 & chr4 & NSG1 \\
\hline \multirow[t]{2}{*}{$\mathrm{FSI} \mathrm{C}^{\mathrm{C}}$} & $\operatorname{cg} 24715106$ & 0.54 & 2.51E-07 & 0.2 & chr11 & AQP11 \\
\hline & $\operatorname{cg} 23782719$ & -0.42 & $2.54 \mathrm{E}-06$ & 0.56 & chr6 & RNF182 \\
\hline \multicolumn{7}{|c|}{$\begin{array}{l}\text { The table shows top two CpGs from the EWAS of the interaction analysis that are associated with the } \\
\text { stress measures. }\end{array}$} \\
\hline \multicolumn{7}{|c|}{${ }^{\mathrm{a} C}$ Cohen perceived stress scale } \\
\hline \multicolumn{7}{|c|}{${ }^{\mathrm{b}}$ Prenatal distress questionnaire } \\
\hline \multicolumn{7}{|l|}{${ }^{\mathrm{C}}$ Fetal stress Index } \\
\hline \multicolumn{7}{|c|}{${ }^{\mathrm{d}}$ Regression coefficients from the statistical model } \\
\hline \multicolumn{7}{|c|}{ eSignificance from the statistical model } \\
\hline \multicolumn{7}{|c|}{${ }^{f}$ False discovery rate } \\
\hline \multicolumn{7}{|l|}{${ }^{\text {g}}$ Chromosome } \\
\hline NA: Not available & & & & & & \\
\hline
\end{tabular}

Network analysis of DMPs

In a second layer of analysis, the network interactions between the proteins encoded by the genes that were annotated to significant DMPs were analyzed using STRING-db, a database and software application enabling an semi-unsupervised statistical network analysis of known and predicted proteinprotein interactions as well as their physical and functional interaction networks based on computational predictions, i.e., enrichment (25). Unique URL for the resulting analysis is as follows:

https://version-11-5.string-db.org/cgi/network?taskld=bMnf9hlgSYrT\&sessionld=bl9Pry04KzyB . 
The top three biological processes identified were 1) Hippo signaling pathway, 2) Regulation of canonical Wnt signaling pathway and 3) Cell- cell junction assembly. Figure 5 shows YAP1 interacting with several proteins of the Hippo-signaling pathway and the ß-Catenin signaling pathways-CTNNB1 (Catenin Beta 1). YAP appears also directly related to TEAD1 and TEAD4 since YAP/TAZ are transcriptional coregulators and partners of the TEAD family transcription factors.

The Kyoto Encyclopedia of Genes and Genomes (KEGG) pathways map molecular objects such as genes, proteins etc. to molecular interactions or relations. The top pathway identified from the KEGG pathways was the Hippo signaling pathway.

As the next step, we used the SFARI gene database(26) to extract information for the genes annotated to CpGs specific to ASD(27). SFARI gene is a database centered on genes involved in autism and has up-todate information on all human genes associated with ASD. Of all the genes looked up in this database (26), only CSMD1 appeared with a score of three indicating strong relevance to ASD (gene.sfari.org/database/human-gene/CSMD1).

\section{Differentially methylated regions (DMRs)}

DMRs are genomic regions that have consistently different DNA methylation across multiple adjacent CpGs (28). The DMRs mapped to or near the genes that are enriched for the biological process of the regulation of sequence-specific DNA binding transcription factor activity suggest that these genes are involved in regulation of gene expression. Regional analysis identified associations with maternal stress measures (PDQ and Cortisol). All DMRs identified by DMRcate as well as the DMPs overlapped with the DMRs identified by comb-p. Results are shown in Table 4.

DMRcate identified two DMRs related to maternal stress measures PDQ and cortisol. One DMR associated with PDQ was found to be located in the DAXX locus (Death-associated protein 6) on chromosome 6. The other DMR, associated with cortisol, was found to be located in the ARL4D (ADPRibosylation factor 4D) on chromosome 17. 
Table 4

Differentially methylated regions (DMRs) in salivary DNA associated with stress measures in FELICITy study.

\begin{tabular}{|c|c|c|c|c|c|c|c|}
\hline Stress measures & $\mathrm{Chr}^{\mathrm{b}}$ & Start $\left(b^{c}\right)^{c}$ & End(bp) & $\mathrm{CpGs}^{\mathrm{d}}$ & p-value $e^{e}$ & Sidak $\mathrm{P}^{f}$ & Gene \\
\hline $\mathrm{PDQ}^{\mathrm{a}}$ & 6 & 33288180 & 33288600 & 8 & 0.000166981 & 8.67E-08 & DAXX \\
\hline Cortisol & 17 & 41476044 & 41476457 & 11 & $6.50 \mathrm{E}-10$ & 1.27E-06 & ARL4D \\
\hline \multicolumn{8}{|c|}{ Marked in bold are significant. } \\
\hline \multicolumn{8}{|c|}{ aprenatal distress questionnaire } \\
\hline \multicolumn{8}{|l|}{${ }^{\mathrm{b}}$ Chromosome } \\
\hline \multicolumn{8}{|c|}{ 'Physical position (basepair) } \\
\hline
\end{tabular}

eStatistical significance

${ }^{f} \mathrm{p}$ of Sidak multiple-testing correction

\section{Discussion}

Most of the maternal stress studies in the past have been limited to targeted DNA methylation analyses in candidate genes $(29,30)$. It is only in the recent years that epigenome wide studies of DNA methylation have gained popularity allowing to evaluate locus-specific methylation across the entire genome(31-34). These different approaches have been recently summarized in two reviews and a meta-analysis $(18,35$, 36). When analyzing these studies, a general conclusion on what type of epigenetic signature is observed in prenatally stressed infants is difficult to draw since many methodological differences are still observed in terms of the type and timing of the prenatal insult studied, the age of the child and the tissue employed to detect DNA methylation. This makes comparison among studies very difficult leading to inconclusive evidence on the association between PS and DNA methylation in the neonate. To bridge this gap, we have examined the association between psychological, molecular and biophysical maternal-fetal stress measures and the genome wide methylation profile in newborn saliva. Our findings validate the hypothesis that PS biomarkers are associated with epigenome-wide DNA methylation in newborn saliva across multiple $\mathrm{CpG}$ sites, in particular, those relevant to neuronal, immune and endocrine homeostasis.

\section{Maternal stress measures and DNA methylation patterns}

In our study, PDQ, but not PSS, and cortisol showed a significant association with five CpG sites. Out of these five CpGs, three were annotated to YAP1, TOMM20 and CSMD1; two CpGs were not annotated to any gene but lay within 50kb of SSBP4, SCAMP1 and LRRC25. We discuss the functional implications of these associations in the following paragraphs. 
YAP1 and its related protein WWdomain- containing transcription regulator 1 (WWTR1; also known as TAZ) (YAP/TAZ) are the main effectors of the Hippo signaling pathway (37). This evolutionarily conserved signaling cascade regulates cell proliferation, stemness, organ size control, and regeneration. Its dysregulation has been associated to multiple forms of cancers, the immunity response and cardiovascular diseases $(37,38)$. Although widely expressed in several tissues, YAP is selectively expressed in astrocytes and neural stem cells in the mouse developing brain and its deletion causes reactive astrogliosis and astrocyte-driven microglial activation (39). Moreover, Passaro et al (40),demonstrated that the transient downregulation of YAP in mouse embryonic stem cells disrupts cellular homeostasis altering the ability to differentiate properly. In our study the hypermethylated CpG cg06542869 annotated to YAP1 is associated with specific pregnancy worries (PDQ score). The functional consequence of the hypermethylation of one single $\mathrm{CpG}$ site in the open sea of the YAP1 gene is highly speculative without evaluating the translated protein. However, it has been demonstrated that the methylation of one single $\mathrm{CpG}$ can impact on the methylation levels of neighboring $\mathrm{CpG}$ sites (41). Assuming that hypermethylation is generally associated with transcriptional silencing of genes, the modification of methylation status of the $Y A P 1$ gene might potentially lead to alterations in cell proliferation, cell differentiation and astrogliosis. In fact, the network analysis of the protein encoded by YAP1 using STRING-db showed an interaction with several proteins of the Hippo signaling pathway such as the TEAD family of transcription proteins. The phosphorylation and inhibition of YAP/TAZ activate the Hippo pathway limiting tissue growth and cell proliferation. Upon dephosphorylation, YAP/TAZ translocate to the nucleus, binding to TEAD and inducing transcriptional programs related to cell proliferation, survival, and migration (37).

TOMM20 (Translocase of outer mitochondrial membrane 20) is involved in glucose/ energy metabolism and deubiquitination. Together with TOMM22 functions as a transit peptide receptor at the surface of the mitochondrial outer membrane and facilitates the movement of preproteins $(42,43)$. Diseases associated with TOMM20 include Optic Atrophy 1 and 11. Our results show that the hypermethylated $\mathrm{CpG}$ site cg25252839 is associated with cortisol levels and annotates to TOMM20.

The CSMD1 gene has been proposed to have brain specificity since it encodes a cell adhesion molecule highly expressed in membrane associated proteins in the CNS, with almost no detection in other tissues (44). The CSMD1 protein is related to immune function playing a crucial role in regulating complement activation and inflammation in the developing brain $(44,45)$ and may also play a role in growth cone function (46). The CSMD1 protein is predominantly expressed in neurons mainly in the cerebral cortex and the hippocampus and has been involved in brain circuits development, neurotransmission, axon guidance, regeneration and plasticity (44). CSMD1 protein coding gene has been previously associated to Autism Spectrum Disorders (ASD) $(47,48)$. Corroborating the above statement, CSMD1 also appears on the SFARI database listing genes associated with ASD. It scored as level 3, meaning there is suggestive evidence from significant but non-replicated association studies. Moreover, CSMD1 has been associated to posttraumatic stress disorder $(49,50)$, schizophrenia $(44,45,51,52)$, and bipolar disorders $(53)$. 
In our study, we found that the hypo-methylated CpG cg05306225 annotates for the gene CSMD1 and is associated with high maternal cortisol levels. Although it is difficult to predict the functional consequences of this single site hypomethylation as mentioned above, it is interesting to observe that the probable destabilization of the methylation status of flanking $\mathrm{CpGs}$ mentioned before, is in a gene with high brain specificity and associated with several neuropsychiatric disorders. In particular, the association of this gene with ASD refers back to several reports showing that the risk for ASD is linked to PS $(11,54)$.

The two other $\mathrm{CpGs}$ that were significantly associated with cortisol levels but are not annotated to any gene are cg11409463 and cg20905655, both hypermethylated. The nearest gene to the CpG site cg11409463 is SCAMP1 whose protein is involved in secretion and transportation. Diseases associated with this gene include Childhood Kidney Cell Carcinoma and Branchiootorenal Syndrome 1. This same CpG site overlaps with several transcription factor binding sites from the AP-1 family (the Jun, the Fos and ATF-2 subfamily) such as JUNB, FOS, SETDB1, ATF3, CBX3, TRIM28, ZNF143. The AP-1 family is responsible for cell growth, differentiation (55) and apoptosis (56). The nearest genes to CpG site cg20905655 are SSBP4 and LRRC25, the latter related to autophagic degradation. So far, not much is known about the functional role of SSB4 and its relation to stress yet.

Of interest, cortisol-associated methylation disbalances in several genes found in neonatal saliva suggest that the transplacental barrier might be impaired and abnormally permeable to steroid hormones. In fact, it has been described that the metabolizing enzymes that lay within trophoblasts and protect the fetus from overexposure to glucocorticoids, are sensitive to maternal stress $(57,58)$. For example, the glucocorticoid-inactivating enzyme, $11 \beta$-hydroxysteroid dehydrogenase type-2 (11ßHSD2), showed a reduced placental expression in relation to maternal anxiety and depressed mood in humans $(59,60)$. The reduced placental expression of $11 \beta \mathrm{HSD} 2$ will potentially lead to a fetal glucocorticoid overexposure affecting developmental events such as fetal growth restriction, altered HPA axis development, impaired offspring brain function, permanent changes in the expression of specific transcription factors and early development of proliferative neural precursors $(57,61)$. Our observation that the newborn saliva shows cortisol associated epigenetic changes in genes related to energy metabolism, cell differentiation and function of the developing brain might be highlighting that one of the underlying mechanisms linking maternal stress with childhood outcomes is through transplacental mediated methylation disbalances in specific genes, among other mechanisms, such as transcriptional regulation of placental gene expression as suggested by Aushev et al (62).

To expand the search of epigenetic signatures associated with stress measures during pregnancy we considered DMRs. Two DMRs were detected, one associated to PDQ (DAXX) and the other to cortisol (ARL4D). DAXX gene encodes for a protein that resides in multiple locations in the nucleus and cytoplasm. Pathways related to Daxx are apoptosis and survival caspase cascade as well as TGF- $\beta$ signalling pathways (63). Diseases associated with DAXX include Gastric neuroendocrine neoplasm, intellectual disability and Alpha-Thalassemia. Interestingly, ATRX gene which has been previously linked with ASD, interacts with DAXX in histone chaperone complex and influences DNA methylation (64-66). Moreover, DAXX is known to be an extended Class II, non-antigen binding HLA (human leukocyte antigen) 
gene associated with autoimmune diseases that interacts with death receptor Fas related to ASD (67). ARL4D belongs to ADP-ribosylation factors (ARFs), members of the Ras family of small GTPases, involved in membrane transport, membrane lipid modifications and maintenance of organelle integrity (68). Interestingly, the transcription of Arl $4 \mathrm{~d}$ was found to be consistently regulated by glucocorticoids such as cortisol (69). So far, not much is known about its function but it has been shown that ArI4D is involved in neurite growth (70), adipogenesis (71) and actin remodeling (72). In adult mice, $A r / 4 d$ is expressed in neocortical layer 1 and hippocampus, mostly in cortical interneurons (CIN), whose loss or alteration have been related to neurological disorders such as autism, schizophrenia, and epilepsy (73). Interestingly, both DMRs are directly or indirectly related to neurological disorders such as ASD. To the best of our knowledge, this is the first report showing significant DMRs in the PS context in newborn saliva samples. Previously Drzymalla et al (74), have identified DMRs related to maternal stress but using cord blood.

Previous studies employing EPIC array on neonatal tissues in association with maternal stress and/or anxiety are limited to one study by Kallak et al,(75). These authors investigated DNA methylation in cord blood of newborns exposed to maternal depression and anxiety. They found two DMPs: one upstream of the ATP Binding Cassette Subfamily F Member 1 gene (ABCF1) and the other upstream of Homo sapiens integrator complex subunit 10 gene (INTS10). Although the maternal stress model is different from ours, it is interesting to note that ABCF1 was previously associated with ASD in a multi-omics data analysis(76). Other comparable studies employing Illumina Infinium 450 BeadChip found mismatching results when studying DNA methylation in infant tissues in relation to maternal stress. Rijlaarsdam et al., (77) showed no associations between PS exposure and neonatal cord blood DNA methylation, whereas Wikenius et al.(78) studying maternal depressive symptoms, found no significant association with 6 weeks infant's saliva DNA methylation. In contrast, Non et al.(33) reported the identification of CpGs located in a cluster of genes related to transcription, translation and cell division processes in cord blood of neonates exposed to non-medicated depression or anxiety.

To summarize these results, we conclude that these genes have been related to several regulatory processes of tissue and cellular homeostasis that, when disturbed, can elicit a stress response (79). Moreover, dysregulation of the expression of CSMD1 and YAP1 have been related to disorders of the immune system as well as of the central and autonomic nervous systems.

\section{Biophysical signature of chronic stress in mother-fetus dyad and DNA methylation}

No significant $\mathrm{CpG}$ sites were observed in association with FSI. This may be either due to insufficient study power or reflect an underlying mechanism. Namely, it is possible that regardless of the non-specific chronic stress perceived by the mother (PSS) and the ANS response of the fetus (FSI), what most impacts the fetal epigenetic profile is the stress generated by specific worries related to pregnancy (captured by PDQ) and the associated high circulating levels of cortisol that is crossing the maternal-fetal placental barrier and impacting the fetal physiology on the scale of epigenome. It is possible that FSI is not the appropriate biophysical correlate of epigenome-level alterations due to PS. In future studies, to 
investigate this relationship further we intend to analyze in more depth the relationships between the neonatal epigenome and the biophysical features of ANS derived from maternal and fetal HRV.

\section{Strengths and Limitations}

In the present study we report the findings of the largest prospectively followed cohort of its kind to date. Several strengths are to be highlighted. Firstly, saliva cells are easy and non-invasive to obtain in newborns. Even though epigenetic changes like DNA methylation are cell and tissue-specific, some CpG sites show cross tissue relevance. Changes in peripheral tissues such as saliva could serve as potential biomarkers for disease risk while also giving an advantage of being non-invasively obtainable. Since the primary organ affected by stress is not available in human studies and post-mortem brain tissue samples cannot capture the fluid state of the epigenome (80), more accessible samples such as saliva and blood are often used as substitutes. Binder and colleagues showed that saliva reflects better DNA methylation patterns of the brain than methylation in blood, highlighting that saliva is the sample medium of choice for epigenetic studies of psychiatric traits, especially in small children (81).

Secondly, we believe that our study's findings can be generalized to the population of pregnant women in most clinics, as this study includes mothers experiencing typical daily stress situations rather than extreme stress exposures.

Limitations to our study are as follows. Our study has a relatively small sample size, which makes identifying subtle differences in methylation difficult. Since there is no other available study with cohorts of pregnant women and newborn saliva samples obtained, we have not yet been able to verify our findings in an independent cohort. Thus, the novel findings of DMPs and DMRs related to these stress measures should be considered as hypothesis-generating and requiring further validation in larger cohorts.

Assessing the DNA methylation levels as soon as the baby is born in association with four stress measures shows the impact of maternal stress on epigenetic marks during the fetal life. However, to serve as early neurodevelopmental biomarkers these marks have to be related to the corresponding neurodevelopmental appraisals. Since epigenetic marks are not fixed at birth and methylation patterns change with age, we are presently carrying out a longitudinal study in this cohort. The DNA methylation status at two years of age will allow us to detect the epigenetic drift defined as the difference in the DNA methylation status over time (78). Moreover, the 2-years' time point will allow us to test for an association with the neurodevelopmental outcome showing the influence of the environment during the first two years of life on the epigenetic traits and whether the present early neonatal epigenetic differences can serve as biomarkers for early interventions to help restore optimal neurodevelopmental trajectories (23).

\section{Conclusions}

In this study, we identified novel associations between newborn epigenome-wide methylation levels measured non-invasively in saliva and chronic psychosocial stress experienced by the mother during 
pregnancy. The epigenetic changes are mostly related to genes involved in secretion and transportation, nuclear signaling, Hippo signaling pathways, apoptosis, intra-cellular trafficking and neuronal signaling. Most strikingly, we found that both DMP (such as CSMD1) and DMRs (DAXX and ARL4D) are annotated to genes related to neurological disorders such as ASD, PTSD and schizophrenia, pointing out to the potential risk of these children to suffer from these disorders.

Taken together, our findings demonstrate that newborns exposed to chronic stress during gestation show DNA methylation signatures related to neuronal, immune and endocrine homeostases.

\section{Materials And Methods}

\section{Study design}

Women with singleton pregnancies, between ages 18-45 in their third trimester (at least 28 weeks gestation) were recruited at the Department of Obstetrics and Gynecology at "Klinikum rechts der Isar" of the Technische Universität München (TUM). Exclusion criteria were serious placental alterations, fetal malformations, and maternal severe illness during pregnancy or use of recreational drugs (24). Between June 2016 and July 2019, 164 women were recruited. Due to methodological problems with saliva sampling, methylation data was available for 114 subjects.

\section{Stress Measurement}

Maternal stress assessment

\section{a) Psychosocial stress assessment.}

Maternal psychosocial stress was measured using the validated German version of Cohen Perceived Stress Scale (PSS-10) (82). PSS-10 is a widely used psychological instrument to measure non-specific perceived chronic stress and measures the degree to which a situation in a person's life is appraised as stressful. It has been validated in German speaking population and is a quick tool for screening chronic stress among prospective subjects (83). In addition, the validated German version of the Prenatal Distress Questionnaire (PDQ) was also administered to the participants to assess specific pregnancy worries and concerns $(24,84-87)$. PSS score and PDQ score were correlated using the Spearman method in R studio.

\section{b) Hair cortisol assessment.}

After delivery maternal hair strands ( $\sim \mathrm{mm}$ diameter) were collected from the posterior vertex region on the head as close to the scalp as possible. The hair samples were sent to the Department of Biochemistry (Endocrinology section) of the Faculty of Pharmacy and Biochemistry (University of Buenos Aires,

Argentina) for cortisol measurement using an automated chemiluminescent immunoassay. Based on the hair growth rate of $1 \mathrm{~cm}$ per month, the $3 \mathrm{~cm}$ long hair segment reflects the integrated hormone secretion over the three-month period prior to sampling. The cortisol was extracted and measured according to 
Iglesias et al (88). This procedure has been validated with the standard method of mass spectrometry and was patented by University of Buenos Aires (89).

\section{Fetal Stress Assessment}

The detailed fetal assessment is described elsewhere(24). In brief, bivariate PRSA (bPRSA) was used to assess the coupling between maternal ( $\mathrm{mHR}$ ) and fHR resulting in Fetal Stress Index (FSI). The fHR was measured by taECG. Fetal ECG extraction algorithm SAVER (90) was applied to detect the fetal R-peaks and the maternal R-peaks in the taECG separately. With the fetal and maternal R peaks, the fetal and maternal RR interval time series were obtained. Mean $\mathrm{fHR}$ and mean maternal heart rate were calculated. Generally, bPRSA identifies and quantifies the relationship between two simultaneously recorded signals. Here, the two signals are mHR as the trigger signal and fHR as the target signal. FSI measures the response of $f H R$ to decreases in $\mathrm{mHR}$.

\section{Sample and Data acquisition}

Newborn saliva sampling

Immediately after delivery, the midwife obtained the newborn saliva/buccal sample by gently rubbing the gums on both sides with the sponge of the Oracollect-DNA kit (DNA Genotek, Canada) and stored it at room temperature. Throughout the manuscript and for ease of reading we have referred as "saliva" to the sample containing both the saliva fluid plus leukocytes and squamous epithelial cells from the oral cavity (91).

DNA extraction

DNA was extracted from $1 \mathrm{ml}$ saliva samples using the PrepIT kit (DNA Genotek). A $1 \mu \mathrm{l}$ aliquot of the extracted samples was checked on $0.8 \%$ Agarose gel and measured via Nano drop for quality. A $2 \mu l$ aliquot of DNA extract from the samples was used to run PCR to check for the sex of the newborn.

Illumina MethylationEPIC BeadChip Array

Bisulphite conversion of DNA and processing of methylation arrays were accomplished in collaboration with the Institute of Epidemiology (Complex diseases group) at Helmholtz Zentrum Munich. For each sample, 500ng of the extracted salivary DNA was treated with sodium bisulphite using the EZ-DNA methylation kit following the manufacturer's protocol. DNAm was assessed using the Illumina Infinium Human MethylationEPIC BeadChip array (Illumina Inc., San Diego, CA, USA) according to the manufacturer's instructions. This array measures over 850,000 loci at a single nucleotide resolution. The BeadChip includes probe types of two different chemistries: (1) Type I probes, in which two different probe types interrogate each $\mathrm{CpG}$ site, one which targets methylated DNA and one that targets unmethylated DNA. (2) Type II probes binding to the nucleotide just before the target site, and create a single base extension of $\mathrm{G}$ or $\mathrm{A}$ complementary to the methylated $\mathrm{C}$ or unmethylated $\mathrm{T}$. 


\section{Data processing}

Quality control

The arrays were scanned using an Illumina iScan reader and processed using GenomeStudio software (Illumina, Inc.). The raw data (idat files) were imported into R using the Bioconductor minfi package (92) and CpGs that have below-background expression levels in more than six samples were filtered out. Concordance between the reported sex and methylation predicted sex was confirmed. Probes with common single nucleotide polymorphism (SNPs) near the methylation binding site were identified and filtered out. To simplify the analysis, probes were restricted to those on autosomal chromosomes. The remaining probes were background-corrected using the out-of-bound probes (93), and normalized using a functional normalization procedure, which uses two principal components of a set of control probes in order to remove technical variability (94). The $\beta$-values (proportion of methylated probes at each $\mathrm{CpG}$ ) were then converted to M-values (logit base 2 of the $\beta$-values), which were used for all linear modeling. To account for unobserved variability or potential batch effects, models were additionally adjusted with three surrogate variables (SV) that were generated from the M-values, using the Bioconductor 'SVA' package (95). The surrogate variables were used as covariates in the statistical analysis. The final analysis included 808,554 probes. Linear regression was used to examine the associations of each CpG site with stress measures. Probes were considered significantly differentially methylated at a false discovery rate $(\mathrm{FDR})<0.05(96)$.

\section{Covariates}

We adjusted for technical covariates, i.e., Illumina plate factor. Furthermore, we included covariates such as newborn sex, gestational age at birth, maternal smoking, autoimmune diseases and gestational diabetes. The sex of the newborn was obtained from the clinical history of the patient. Gestational age was calculated from the first day of the woman's last menstrual cycle to the date of delivery. Maternal smoking was categorized into two categories: 'never smoked' and 'smoking during pregnancy'. Covariates signifying autoimmune diseases and gestational diabetes were categorized into two categories: 'Yes' and 'No'.

It should be noted that the cellular DNA source of saliva is heterogeneous. While there is much literature dealing with saliva cell type composition in infants, children and adults $(78,91,97)$, to date, there are no studies indicating the cell-mixture composition of the saliva of the newborn. Housemann et al, in 2014 (98) introduced the reference-free cell type method to estimate cell types in tissues such as saliva, placenta and adipose tissue(98), which is closely related to surrogate variable analysis (SVA) (99). We have therefore used SVA directly to estimate for all the unobserved variability including cell types. It has been shown that using SVA increases the biological accuracy and reproducibility by identifying the sources of heterogeneity and correctly accounting for them in the analysis (99). Three surrogate variables that were generated in the quality control step, using the SVA package, were also used as covariates in the main model. 


\section{Data Analysis}

We conducted a series of analyses on the genome wide microarray data, with each technique designed to capture potentially different patterns of DNA methylation. The first analysis conducted was EWAS analysis for the DMPs (CpG or site-by-site regression analysis), which analyzed each CpG site individually. Second, we performed a sex-interaction EWAS analysis for the DMPs to compare methylation patterns in terms of sex. The Illumina database ('IlluminaHumanMethylationEPICanno.ilm10b4.hg19') was primarily used for identifying gene annotations for the significant hits. The UCSC genome browser was used to verify genes identified with Illumina database and, where genes were missing in the Illumina database, was searched to augment genes within 50kb of the $\mathrm{CpG}$ site. Third, the biological exploration and network analysis for each CpG annotating to specific genes was conducted using the online software STRING-DB (25) and SFARI (26). Fourth, we conducted DMR analysis (or regional analysis), which captures an average pattern of DNA methylation among neighboring sites. Figure 6 shows a summary of the methodological study design.

Differentially methylated positions (DMPs) analysis.

Each CpG site or DMP was separately tested for association with exposure to stress. Three sets of EWAS analyses were run to identify CpG sites associated with either PSS, PDQ, FSI or cortisol. All the statistical analyses were done in $\mathrm{R}$ version 3.5.2. We used the following linear regression model in 'limma' $\mathrm{R}$ package to test for DMPs: - DNA methylation $\sim$ Stress measures + Newborn sex + Gestational age + Smoking + Autoimmune diseases + gestational diabetes + Illumina plate factor + SVAs. We visualized the epigenome-wide associations study results using Manhattan plots and quantile-quantile (QQ plots). Genomic inflation factor was calculated for each association. We corrected the p-values for inflation if lambda was above 1.1, using a Bayesian method for estimation of empirical null distribution as implemented in R/Bioconductor package 'bacon' (100).

Sex interaction analysis

Sex interaction analysis was performed in the FELICITy cohort for each CpG/DMP site association with the stress measures. The model was identical to the adjusted model, but with a "Sex * methylation" interaction term (male as a reference sex). Statistical significance threshold was set at FDR $<0.05$.

Biological processes and network analysis of DMPs

To investigate whether specific biological processes and networks are over-represented in our EWAS results, network analyses were performed for the DMPs using STRING-db. The protein encoding genes that were annotated to significant DMPs were analyzed using STRING-DB. STRING-DB is an unsupervised statistical network analysis database that has known proteins and their physical and functional interaction networks (25). We used the KEGG database in STRING-DB to explore whether annotated genes have been related to neurobiological and neuronal processes or diseases. We also used the SFARI gene database (26) to extract information for the genes annotated to CpGs specific to ASD (27). 
Differentially methylated regions (DMRs) analysis

DMRs were initially identified using the 'the Bioconductor DMRcate package (101) and verified using comb-p (102). These packages are consistently reported to have the best sensitivity and highest control of false positive rate when compared to other DMR tools (103). A significant DMR can be detected even if there is no genome-wide significant DMP in the region. DMRcate identifies DMRs from the tunable kernel smoothing process of association signals (101).

DMRcate was used on the results of the limma analysis to test for DMRs. The parameters for DMRcate (lambda $=1000, C=2$ ) were set and a FDR cut-off of 0.05 was used to determine significance. Further Comb-p was used to verify DMRs identified by DMRcate. For comb-p, identified DMRs consisting of at least two probes and having a Sidak-corrected $p$-value $<0.05$ were considered statistically significant (102). DMRs were annotated to gene symbols according to genome assembly (hg19). P-value for each DMR was adjusted for multiple testing with Sidak correction method as implemented by default in the 'comb-p' tool.

\section{Abbreviations}

ADHD

Attention- Deficit Hyperactivity Disorder

ANS

Autonomic Nervous System

ARL $4 D$

ADP- Ribosylation factor 4D

ASD

Autism Spectrum Disorder

CNS

Central Nervous System

CSMD1

CUB And Sushi Multiple Domains 1

CTG

Cardiotocography

$D A X X$

Death- associated protein 6

DMPs

DNA Methylated Positions

DMR

DNA Methylated Regions

ECG

Electrocardiogram

EWAS 
Epigenome Wide Association Study

FHR

Fetal Heart Rate

FSI

Fetal Stress Index

HPA axis

Hypothalamic-Pituitary adrenal axis

MHR

Maternal Heart Rate

PDQ

Prenatal Distress Questionnaire

PRSA

Phase-Rectified Signal Averaging

PS

Prenatal Stress

PSS

Perceived Stress Scale

PTSD

Post- Traumatic Stress Disorder

QQ

Quantile- Quantile

SNPS

Single Nucleotide Polymorphism

SV

Surrogate Variables

TaECG

Transabdominal Electrocardiography

TOMM20

Translocase of Outer Mitochondrial Membrane 20

UCSC

University of California Santa Cruz

YAP1

Yes1 regulated transcription factor

\section{Declarations}

\section{Acknowledgements}

The authors wish to thank Nadine Lindemann for technical assistance with EPIC and all the laboratory procedures. We are also grateful to Dr. Jennifer Kriebel for her initial and valuable guidance for running 
the EPIC and raw data extraction and viewing in Genome studio. We are grateful to all participants of FELICITy Study for their invaluable contributions to these studies, as well as all members of the nursing staff at labor and delivery of the Department of Obstetrics and Gynecology, Klinikum rechts der Isar, Technical University of Munich, Germany.

\section{Author contributions}

RS: conducted the experiments and the study-level data processing, statistical analysis and analyses and drafted the manuscript. RS, SML, MGF \& MCA: data collection management, data analysis, and manuscript writing and editing. CZ \& PZ: patient recruitment and data collection. BF \& DG: cortisol analysis in hair samples. RW \& MW: EPIC methylation profiling advice, data analysis and statistical advice. JWM \& TKB: statistical analysis and advice. SML \& MCA: conceptualized the project, protocol and project development, data collection and management, data analysis and funding acquisition. All authors read and approved the final manuscript.

\section{Funding}

This work was supported by a Hans Fischer Senior Fellowship from IAS-TUM (Institute for Advanced Study- Technical University of Munich, Munich, Germany) awarded to MCA and funds from the Department of Obstetrics and Gynecology, Klinikum rechts der Isar, Technical University of Munich, Germany to SML. This study was also supported by resources from the University of Washington (UW) Interdisciplinary Center for Exposures, Diseases, Genomics and Environment (P30ES007033) and the UW Intellectual and Developmental Disabilities Research Center (P50HD103524) to JWM \& TKB.

\section{Availability of data and materials}

The datasets supporting these findings are not publicly available. Instead, the datasets used and/or analyzed for the current study are available from the corresponding author on reasonable request and after Institutional Review Board review and approval. Scripts used in data processing and statistical analyses have been made publicly accessible at https://ascgitlab.helmholtzmuenchen.de/ritika.sharma/felicity-project/-/tree/master/

\section{Ethics approval and consent to participate}

The study protocol is in strict accordance with the Committee of Ethical Principles for Medical Research from TUM and has the approval of the "Ethikkommission der Fakultät für Medizin der Technische Universität München" (registration number 151/16S). ClinicalTrials.gov registration number is NCT03389178. Written informed consent was received from participants prior to inclusion in the study.

\section{Consent for Publication}


Not applicable.

\section{Competing Interests}

MGF holds a pending U.S. patent on fetal ECG technology (Application \#20210330236). The remaining authors declare that the research was conducted in the absence of any commercial or financial relationships that could be construed as a potential conflict of interest.

\section{Authors' Information}

MCA was awarded with the August Wilhelm Scheer Professorship Program (TUM) twice for a 6 months period stay at the Klinik und Poliklinik für Frauenheilkunde, Technische Universität München, Klinikum rechts der Isar, Munich for the start-up of the FELICITy project together with SML and a Hans Fischer Senior Fellowship (HFSF) from IAS-TUM (Institute for Advanced Study-TUM, Munich). RS holds a Doctoral Fellowship from HFSF/IAS-TUM.

\section{References}

1. Baier CJ, Katunar MR, Adrover E, Pallarés ME, Antonelli MC. Gestational restraint stress and the developing dopaminergic system: an overview. Neurotoxicity research. 2012;22(1):16-32.

2. Bale TL, Baram TZ, Brown AS, Goldstein JM, Insel TR, McCarthy MM, et al. Early life programming and neurodevelopmental disorders. Biological psychiatry. 2010;68(4):314-9.

3. Boersma GJ, Tamashiro KL. Individual differences in the effects of prenatal stress exposure in rodents. Neurobiology of stress. 2015;1:100-8.

4. Brannigan R, Cannon M, Tanskanen A, Huttunen M, Leacy F, Clarke M. The association between subjective maternal stress during pregnancy and offspring clinically diagnosed psychiatric disorders. Acta Psychiatrica Scandinavica. 2019;139(4):304-10.

5. Charil A, Laplante DP, Vaillancourt C, King S. Prenatal stress and brain development. Brain research reviews. 2010;65(1):56-79.

6. Dong E, Pandey SC. Prenatal stress induced chromatin remodeling and risk of psychopathology in adulthood. International review of neurobiology. 2021;156:185.

7. Frasch MG, Lobmaier SM, Stampalija T, Desplats P, Pallarés ME, Pastor V, et al. Non-invasive biomarkers of fetal brain development reflecting prenatal stress: An integrative multi-scale multispecies perspective on data collection and analysis. Neuroscience \& Biobehavioral Reviews. 2020;117:165-83.

8. Rakers F, Rupprecht S, Dreiling M, Bergmeier C, Witte OW, Schwab M. Transfer of maternal psychosocial stress to the fetus. Neuroscience \& Biobehavioral Reviews. 2020;117:185-97. 
9. Monk C, Lugo-Candelas C, Trumpff C. Prenatal developmental origins of future psychopathology: Mechanisms and pathways. Annual review of clinical psychology. 2019;15:317-44.

10. Van den Bergh BR, van den Heuvel MI, Lahti M, Braeken M, de Rooij SR, Entringer S, et al. Prenatal developmental origins of behavior and mental health: The influence of maternal stress in pregnancy. Neuroscience \& Biobehavioral Reviews. 2020;117:26-64.

11. Beversdorf DQ, Manning S, Hillier A, Anderson S, Nordgren R, Walters S, et al. Timing of prenatal stressors and autism. Journal of autism and developmental disorders. 2005;35(4):471-8.

12. Entringer $\mathrm{S}$, Kumsta R, Hellhammer DH, Wadhwa PD, Wüst S. Prenatal exposure to maternal psychosocial stress and HPA axis regulation in young adults. Hormones and behavior. 2009;55(2):292-8.

13. Müller JJ, Antonow-Schlorke I, Kroegel N, Rupprecht S, Rakers F, Witte OW, et al. Cardiovascular effects of prenatal stress-Are there implications for cerebrovascular, cognitive and mental health outcome? Neuroscience \& Biobehavioral Reviews. 2020;117:78-97.

14. Moisiadis VG, Matthews SG. Glucocorticoids and fetal programming part 1: outcomes. Nature Reviews Endocrinology. 2014;10(7):391-402.

15. Monk C, Myers MM, Sloan RP, Ellman LM, Fifer WP. Effects of women's stress-elicited physiological activity and chronic anxiety on fetal heart rate. Journal of Developmental \& Behavioral Pediatrics. 2003;24(1):32-8.

16. Gao Y, Huang Y, Li X. Interaction between prenatal maternal stress and autonomic arousal in predicting conduct problems and psychopathic traits in children. Journal of psychopathology and behavioral assessment. 2017;39(1):1-14.

17. Kinsella MT, Monk C. Impact of maternal stress, depression \& anxiety on fetal neurobehavioral development. Clinical obstetrics and gynecology. 2009;52(3):425.

18. Cao-Lei L, De Rooij S, King S, Matthews S, Metz G, Roseboom T, et al. Prenatal stress and epigenetics. Neuroscience \& Biobehavioral Reviews. 2020;117:198-210.

19. Kundakovic M, Jaric I. The epigenetic link between prenatal adverse environments and neurodevelopmental disorders. Genes. 2017;8(3):104.

20. Bredy TW, Sun YE, Kobor MS. How the epigenome contributes to the development of psychiatric disorders. Developmental psychobiology. 2010;52(4):331-42.

21. Weaver IC, Cervoni N, Champagne FA, D'Alessio AC, Sharma S, Seckl JR, et al. Epigenetic programming by maternal behavior. Nature neuroscience. 2004;7(8):847-54.

22. Wakefield C, Yao L, Self S, Frasch MG. Wearable technology for health monitoring during pregnancy: an observational cross-sectional survey study. medRxiv. 2022:2022.01.26.22269158.

23. Antonelli MC, Frasch MG, Rumi M, Sharma R, Zimmermann P, Molinet MS, et al. Early Biomarkers and Intervention Programs for the Infant Exposed to Prenatal Stress. Current Neuropharmacology. 2020(2022;20(1):):94-106.. 
24. Lobmaier SM, Müller A, Zelgert C, Shen C, Su PC, Schmidt G, et al. Fetal heart rate variability responsiveness to maternal stress, non-invasively detected from maternal transabdominal ECG. Archives of gynecology and obstetrics. 2020;301(2):405-14.

25. Szklarczyk D, Gable AL, Lyon D, Junge A, Wyder S, Huerta-Cepas J, et al. STRING v11: protein-protein association networks with increased coverage, supporting functional discovery in genome-wide experimental datasets. Nucleic acids research. 2019;47(D1):D607-D13.

26. Abrahams BS, Arking DE, Campbell DB, Mefford HC, Morrow EM, Weiss LA, et al. SFARI Gene 2.0: a community-driven knowledgebase for the autism spectrum disorders (ASDs). Molecular autism. 2013;4(1):1-3.

27. MG Frasch GS, MC Antonelli. Autism spectrum disorder: a neuro-immunometabolic hypothesis of the developmental origins" Journal of Developmental Origins of Health and Disease 2019.

28. Rakyan VK, Down TA, Balding DJ, Beck S. Epigenome-wide association studies for common human diseases. Nature Reviews Genetics. 2011;12(8):529-41.

29. Monk C, Feng T, Lee S, Krupska I, Champagne FA, Tycko B. Distress during pregnancy: epigenetic regulation of placenta glucocorticoid-related genes and fetal neurobehavior. American Journal of Psychiatry. 2016;173(7):705-13.

30. Oberlander TF, Weinberg J, Papsdorf M, Grunau R, Misri S, Devlin AM. Prenatal exposure to maternal depression, neonatal methylation of human glucocorticoid receptor gene (NR3C1) and infant cortisol stress responses. Epigenetics. 2008;3(2):97-106.

31. Cao-Lei L, Massart R, Suderman MJ, Machnes Z, Elgbeili G, Laplante DP, et al. DNA methylation signatures triggered by prenatal maternal stress exposure to a natural disaster: Project Ice Storm. PloS one. 2014;9(9):e107653.

32. Mehta D, Klengel T, Conneely KN, Smith AK, Altmann A, Pace TW, et al. Childhood maltreatment is associated with distinct genomic and epigenetic profiles in posttraumatic stress disorder. Proceedings of the national academy of sciences. 2013;110(20):8302-7.

33. Non AL, Binder AM, Kubzansky LD, Michels KB. Genome-wide DNA methylation in neonates exposed to maternal depression, anxiety, or SSRI medication during pregnancy. Epigenetics. 2014;9(7):96472.

34. Tobi EW, Slieker RC, Stein AD, Suchiman HED, Slagboom PE, Van Zwet EW, et al. Early gestation as the critical time-window for changes in the prenatal environment to affect the adult human blood methylome. International journal of epidemiology. 2015;44(4):1211-23.

35. Sammallahti S, Hidalgo APC, Tuominen S, Malmberg A, Mulder RH, Brunst KJ, et al. Maternal anxiety during pregnancy and newborn epigenome-wide DNA methylation. Molecular Psychiatry. 2021:1-14.

36. Sosnowski DW, Booth C, York TP, Amstadter AB, Kliewer W. Maternal prenatal stress and infant DNA methylation: a systematic review. Developmental psychobiology. 2018;60(2):127-39.

37. Ma S, Meng Z, Chen R, Guan K-L. The Hippo pathway: biology and pathophysiology. Annual review of biochemistry. 2019;88:577-604. 
38. Kandilya D, Shyamasundar S, Singh DK, Banik A, Hande MP, Stünkel W, et al. High glucose alters the DNA methylation pattern of neurodevelopment associated genes in human neural progenitor cells in vitro. Scientific reports. 2020;10(1):1-14.

39. Huang Z, Wang Y, Hu G, Zhou J, Mei L, Xiong W-C. YAP is a critical inducer of SOCS3, preventing reactive astrogliosis. Cerebral cortex. 2016;26(5):2299-310.

40. Passaro F, De Martino I, Zambelli F, Di Benedetto G, Barbato M, D'Erchia AM, et al. YAP contributes to DNA methylation remodeling upon mouse embryonic stem cell differentiation. Journal of Biological Chemistry. 2021;296.

41. Vohra M, Sharma AR, Rai PS. SNPs in sites for DNA methylation, transcription factor binding, and miRNA targets leading to allele-specific gene expression and contributing to complex disease risk: a systematic review. Public Health Genomics. 2020:1-16.

42. Hernández JM, Giner P, Hernández-Yago J. Gene structure of the human mitochondrial outer membrane receptor Tom 20 and evolutionary study of its family of processed pseudogenes. Gene. 1999;239(2):283-91.

43. Swie Goping I, Millar DG, Shore GC. Identification of the human mitochondrial protein import receptor, huMas20p. Complementation of $\Delta$ mas20 in yeast. FEBS letters. 1995;373(1):45-50.

44. Abd El Gayed EM, Rizk MS, Ramadan AN, Bayomy NR. mRNA Expression of the CUB and Sushi Multiple Domains 1 (CSMD1) and Its Serum Protein Level as Predictors for Psychosis in the Familial High-Risk Children and Young Adults. ACS omega. 2021;6(37):24128-38.

45. Liu Y, Fu X, Tang Z, Li C, Xu Y, Zhang F, et al. Altered expression of the CSMD1 gene in the peripheral blood of schizophrenia patients. BMC psychiatry. 2019;19(1):1-5.

46. Kraus DM, Elliott GS, Chute H, Horan T, Pfenninger KH, Sanford SD, et al. CSMD1 is a novel multiple domain complement-regulatory protein highly expressed in the central nervous system and epithelial tissues. The Journal of Immunology. 2006;176(7):4419-30.

47. Cukier HN, Dueker ND, Slifer SH, Lee JM, Whitehead PL, Lalanne E, et al. Exome sequencing of extended families with autism reveals genes shared across neurodevelopmental and neuropsychiatric disorders. Molecular autism. 2014;5(1):1-10.

48. Guo H, Peng Y, Hu Z, Li Y, Xun G, Ou J, et al. Genome-wide copy number variation analysis in a Chinese autism spectrum disorder cohort. Scientific reports. 2017;7(1):1-9.

49. Melroy-Greif WE, Wilhelmsen KC, Yehuda R, Ehlers CL. Genome-wide association study of posttraumatic stress disorder in two high-risk populations. Twin Research and Human Genetics. 2017;20(3):197-207.

50. Nievergelt CM, Maihofer AX, Mustapic M, Yurgil KA, Schork NJ, Miller MW, et al. Genomic predictors of combat stress vulnerability and resilience in US Marines: a genome-wide association study across multiple ancestries implicates PRTFDC1 as a potential PTSD gene. Psychoneuroendocrinology. 2015;51:459-71.

51. Consortium SPG-WAS. Genome-wide association study identifies five new schizophrenia loci. Nature genetics. 2011;43(10):969. 
52. Håvik B, Le Hellard S, Rietschel M, Lybæk H, Djurovic S, Mattheisen M, et al. The complement controlrelated genes CSMD1 and CSMD2 associate to schizophrenia. Biological psychiatry. 2011;70(1):3542.

53. Xu W, Cohen-Woods S, Chen Q, Noor A, Knight J, Hosang G, et al. Genome-wide association study of bipolar disorder in Canadian and UK populations corroborates disease loci including SYNE1 and CSMD1. BMC medical genetics. 2014;15(1):1-13.

54. Kinney DK, Munir KM, Crowley DJ, Miller AM. Prenatal stress and risk for autism. Neuroscience \& Biobehavioral Reviews. 2008;32(8):1519-32.

55. Angel P, Karin M. The role of Jun, Fos and the AP-1 complex in cell-proliferation and transformation. Biochimica et Biophysica Acta (BBA)-Reviews on Cancer. 1991;1072(2-3):129-57.

56. Ameyar M, Wisniewska M, Weitzman J. A role for AP-1 in apoptosis: the case for and against. Biochimie. 2003;85(8):747-52.

57. Seckl JR, Holmes MC. Mechanisms of disease: glucocorticoids, their placental metabolism and fetal'programming'of adult pathophysiology. Nature clinical practice Endocrinology \& metabolism. 2007;3(6):479-88.

58. Aye IL, Keelan JA. Placental ABC transporters, cellular toxicity and stress in pregnancy. Chemicobiological interactions. 2013;203(2):456-66.

59. Jensen Peña C, Monk C, Champagne FA. Epigenetic effects of prenatal stress on $11 \beta$-hydroxysteroid dehydrogenase-2 in the placenta and fetal brain. PloS one. 2012;7(6):e39791.

60. O'Donnell KJ, Jensen AB, Freeman L, Khalife N, O'Connor TG, Glover V. Maternal prenatal anxiety and downregulation of placental 11ß-HSD2. Psychoneuroendocrinology. 2012;37(6):818-26.

61. Shams M, Kilby M, Somerset D, Howie A, Gupta A, Wood P, et al. 11Beta-hydroxysteroid dehydrogenase type 2 in human pregnancy and reduced expression in intrauterine growth restriction. Human Reproduction (Oxford, England). 1998;13(4):799-804.

62. Aushev VN, Li Q, Deyssenroth M, Zhang W, Finik J, Hurd YL, et al. Placental gene network modules are associated with maternal stress during pregnancy and infant temperament. The FASEB Journal. 2021;35(10):e21922.

63. Yang X, Khosravi-Far R, Chang HY, Baltimore D. Daxx, a novel Fas-binding protein that activates JNK and apoptosis. Cell. 1997;89(7):1067-76.

64. Fitzgerald T, Gerety S, Jones W, Van Kogelenberg M, King D, McRae J, et al. Large-scale discovery of novel genetic causes of developmental disorders. Nature. 2015;519(7542):223.

65. Tremblay MW, Jiang Y-h. DNA methylation and susceptibility to autism spectrum disorder. Annual review of medicine. 2019;70:151-66.

66. Hoelper D, Huang H, Jain AY, Patel DJ, Lewis PW. Structural and mechanistic insights into ATRXdependent and-independent functions of the histone chaperone DAXX. Nature communications. 2017;8(1):1-13. 
67. Torres AR, Westover JB, Rosenspire AJ. HLA immune function genes in autism. Autism research and treatment. 2012;2012.

68. D'Souza-Schorey C, Chavrier P. ARF proteins: roles in membrane traffic and beyond. Nature reviews Molecular cell biology. 2006;7(5):347-58.

69. Juszczak GR, Stankiewicz AM. Glucocorticoids, genes and brain function. Progress in NeuroPsychopharmacology and Biological Psychiatry. 2018;82:136-68.

70. Yamauchi J, Miyamoto Y, Torii T, Mizutani R, Nakamura K, Sanbe A, et al. Valproic acid-inducible Arl4D and cytohesin-2/ARNO, acting through the downstream Arf6, regulate neurite outgrowth in N1E-115 cells. Experimental cell research. 2009;315(12):2043-52.

71. Yu J, Ka S-O, Kwon K-B, Lee S-M, Park J-W, Park B-H. Overexpression of the small GTPase ArI4D suppresses adipogenesis. International journal of molecular medicine. 2011;28(5):793-8.

72. Li C-C, Chiang T-C, Wu T-S, Pacheco-Rodriguez G, Moss J, Lee F-JS. ARL4D recruits cytohesin2/ARNO to modulate actin remodeling. Molecular biology of the cell. 2007;18(11):4420-37.

73. Rubin AN, Malik R, Cho KK, Lim KJ, Lindtner S, Schwartz SER, et al. Regulatory elements inserted into AAVs confer preferential activity in cortical interneurons. ENeuro. 2020;7(6).

74. Drzymalla E, Gladish N, Koen N, Epstein MP, Kobor MS, Zar HJ, et al. Association between maternal depression during pregnancy and newborn DNA methylation. Translational psychiatry. 2021;11(1):18.

75. Kallak TK, Bränn E, Fransson E, Johansson Å, Lager S, Comasco E, et al. DNA methylation in cord blood in association with prenatal depressive symptoms. Clinical epigenetics. 2021;13(1):1-14.

76. Sun Y, Yao X, March ME, Meng X, Li J, Wei Z, et al. Target genes of autism risk loci in brain frontal cortex. Frontiers in genetics. 2019;10:707.

77. Rijlaarsdam J, Pappa I, Walton E, Bakermans-Kranenburg MJ, Mileva-Seitz VR, Rippe RC, et al. An epigenome-wide association meta-analysis of prenatal maternal stress in neonates: A model approach for replication. Epigenetics. 2016;11(2):140-9.

78. Wikenius E, Myhre AM, Page CM, Moe V, Smith L, Heiervang ER, et al. Prenatal maternal depressive symptoms and infant DNA methylation: a longitudinal epigenome-wide study. Nordic journal of psychiatry. 2019;73(4-5):257-63.

79. Chovatiya R, Medzhitov R. Stress, inflammation, and defense of homeostasis. Molecular cell. 2014;54(2):281-8.

80. Tylee DS, Kawaguchi DM, Glatt SJ. On the outside, looking in: A review and evaluation of the comparability of blood and brain "-omes". American Journal of Medical Genetics Part B: Neuropsychiatric Genetics. 2013;162(7):595-603.

81. Smith AK, Kilaru V, Klengel T, Mercer KB, Bradley B, Conneely KN, et al. DNA extracted from saliva for methylation studies of psychiatric traits: evidence tissue specificity and relatedness to brain. American Journal of Medical Genetics Part B: Neuropsychiatric Genetics. 2015;168(1):36-44. 
82. Cohen S, Kamarck T, Mermelstein R. A global measure of perceived stress. Journal of health and social behavior. 1983:385-96.

83. Klein EM, Brähler E, Dreier M, Reinecke L, Müller KW, Schmutzer G, et al. The German version of the Perceived Stress Scale-psychometric characteristics in a representative German community sample. BMC psychiatry. 2016;16(1):1-10.

84. Alderdice F, Lynn F. Factor structure of the prenatal distress questionnaire. Midwifery. 2011;27(4):553-9.

85. Caparros-Gonzalez RA, Perra O, Alderdice F, Lynn F, Lobel M, García-García I, et al. Psychometric validation of the Prenatal Distress Questionnaire (PDQ) in pregnant women in Spain. Women \& health. 2019;59(8):937-52.

86. Lobmaier SM, Müller A, Zelgert C, Shen C, Su P, Schmidt G, et al. Fetal heart rate variability responsiveness to maternal stress, non-invasively detected from maternal transabdominal ECG. Archives of gynecology and obstetrics. 2020;301(2):405-14.

87. Zimmermann P, Antonelli MC, Sharma R, Müller A, Zelgert C, Fabre B, et al. Prenatal stress perturbs neonatal iron homeostasis in a sex-specific manner. arXiv preprint arXiv:210512809. 2021.

88. Iglesias S, Jacobsen D, Gonzalez D, Azzara S, Repetto EM, Jamardo J, et al. Hair cortisol: A new tool for evaluating stress in programs of stress management. Life sciences. 2015;141:188-92.

89. Gonzalez D, Jacobsen D, Ibar C, Pavan C, Monti J, Machulsky NF, et al. Hair cortisol measurement by an automated method. Scientific reports. 2019;9(1):1-6.

90. Li R, Frasch MG, Wu H-T. Efficient fetal-maternal ECG signal separation from two channel maternal abdominal ECG via diffusion-based channel selection. Frontiers in physiology. 2017;8:277.

91. Theda C, Hwang SH, Czajko A, Loke YJ, Leong P, Craig JM. Quantitation of the cellular content of saliva and buccal swab samples. Scientific reports. 2018;8(1):1-8.

92. Aryee MJ, Jaffe AE, Corrada-Bravo H, Ladd-Acosta C, Feinberg AP, Hansen KD, et al. Minfi: a flexible and comprehensive Bioconductor package for the analysis of Infinium DNA methylation microarrays. Bioinformatics. 2014;30(10):1363-9.

93. Triche Jr TJ, Weisenberger DJ, Van Den Berg D, Laird PW, Siegmund KD. Low-level processing of Illumina Infinium DNA methylation beadarrays. Nucleic acids research. 2013;41(7):e90-e.

94. Fortin J-P, Labbe A, Lemire M, Zanke BW, Hudson TJ, Fertig EJ, et al. Functional normalization of 450k methylation array data improves replication in large cancer studies. Genome biology. 2014;15(11):1-17.

95. Leek JT, Johnson WE, Parker HS, Jaffe AE, Storey JD. The sva package for removing batch effects and other unwanted variation in high-throughput experiments. Bioinformatics. 2012;28(6):882-3.

96. Benjamini Y, Hochberg Y. Controlling the false discovery rate: a practical and powerful approach to multiple testing. Journal of the Royal statistical society: series B (Methodological). 1995;57(1):289300 . 
97. Van Dongen J, Ehli EA, Jansen R, Van Beijsterveldt CE, Willemsen G, Hottenga JJ, et al. Genome-wide analysis of DNA methylation in buccal cells: a study of monozygotic twins and mQTLs. Epigenetics \& chromatin. 2018;11(1):1-14.

98. Houseman EA, Molitor J, Marsit CJ. Reference-free cell mixture adjustments in analysis of DNA methylation data. Bioinformatics. 2014;30(10):1431-9.

99. Leek JT, Storey JD. Capturing heterogeneity in gene expression studies by surrogate variable analysis. PLoS genetics. 2007;3(9):e161.

100. van Iterson M, van Zwet EW, Heijmans BT. Controlling bias and inflation in epigenome-and transcriptome-wide association studies using the empirical null distribution. Genome biology. 2017;18(1):1-13.

101. Peters TJ, Buckley MJ, Statham AL, Pidsley R, Samaras K, Lord RV, et al. De novo identification of differentially methylated regions in the human genome. Epigenetics \& chromatin. 2015;8(1):1-16.

102. Pedersen BS, Schwartz DA, Yang IV, Kechris KJ. Comb-p: software for combining, analyzing, grouping and correcting spatially correlated P-values. Bioinformatics. 2012;28(22):2986-8.

103. Mallik S, Odom GJ, Gao Z, Gomez L, Chen X, Wang L. An evaluation of supervised methods for identifying differentially methylated regions in Illumina methylation arrays. Briefings in bioinformatics. 2019;20(6):2224-35.

\section{Figures}

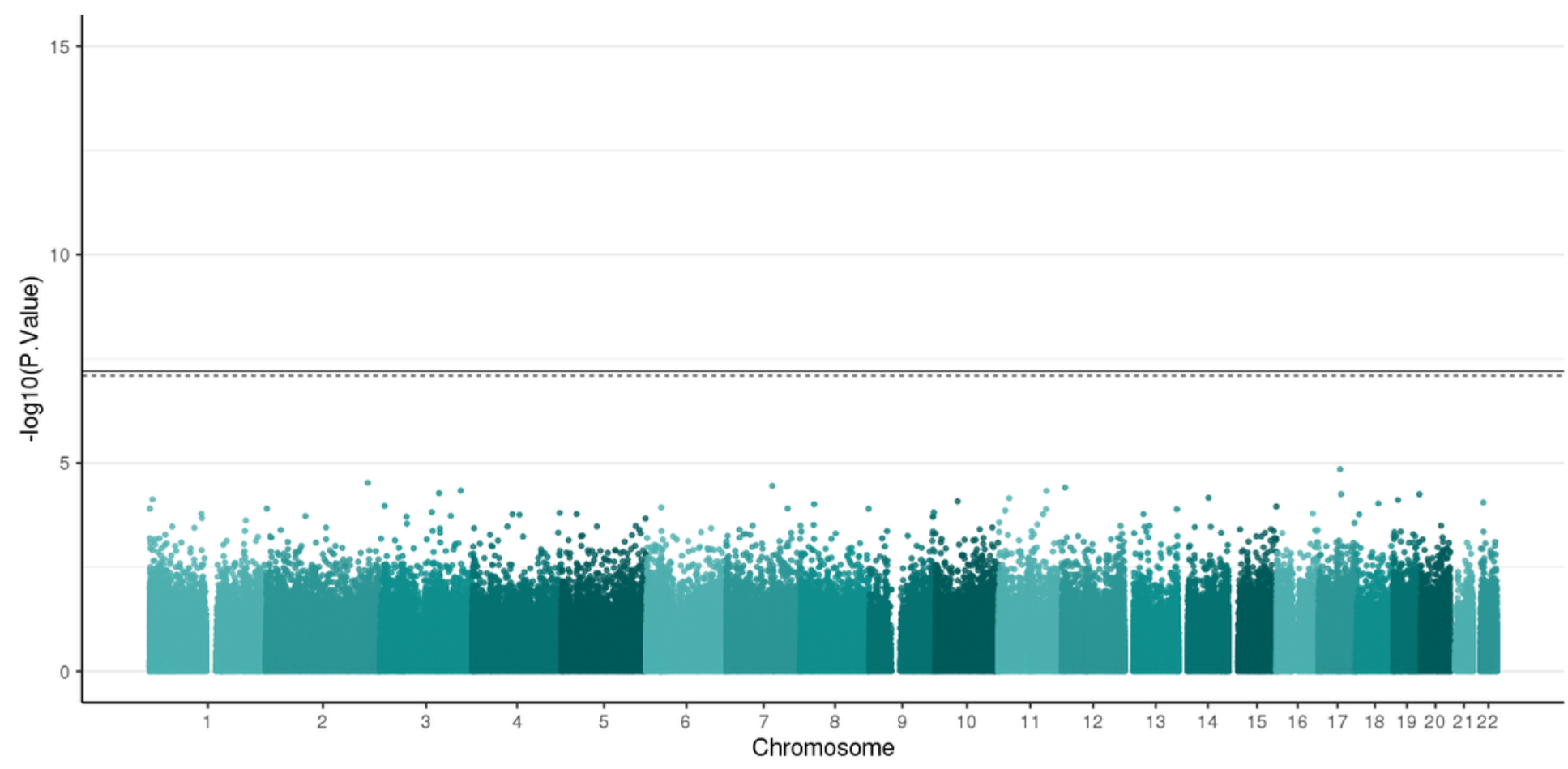

Figure 1

Manhattan plot and Q-Q plot of salivary DNA methylation associated with PSS. 
Manhattan plots of salivary DNA methylation associated with PSS. The x-axis represents the genomic loci of the individual $\mathrm{CpGs}$ and the $y$-axis represents the -log10 (p-value). Black line: Bonferroni threshold $(p=6.183879 \mathrm{e}-08)$ and the dotted line: Multiple testing correction threshold (FDR <0.05) have been added to the plot. There are no $\mathrm{CpGs}$ that cross the significance threshold.

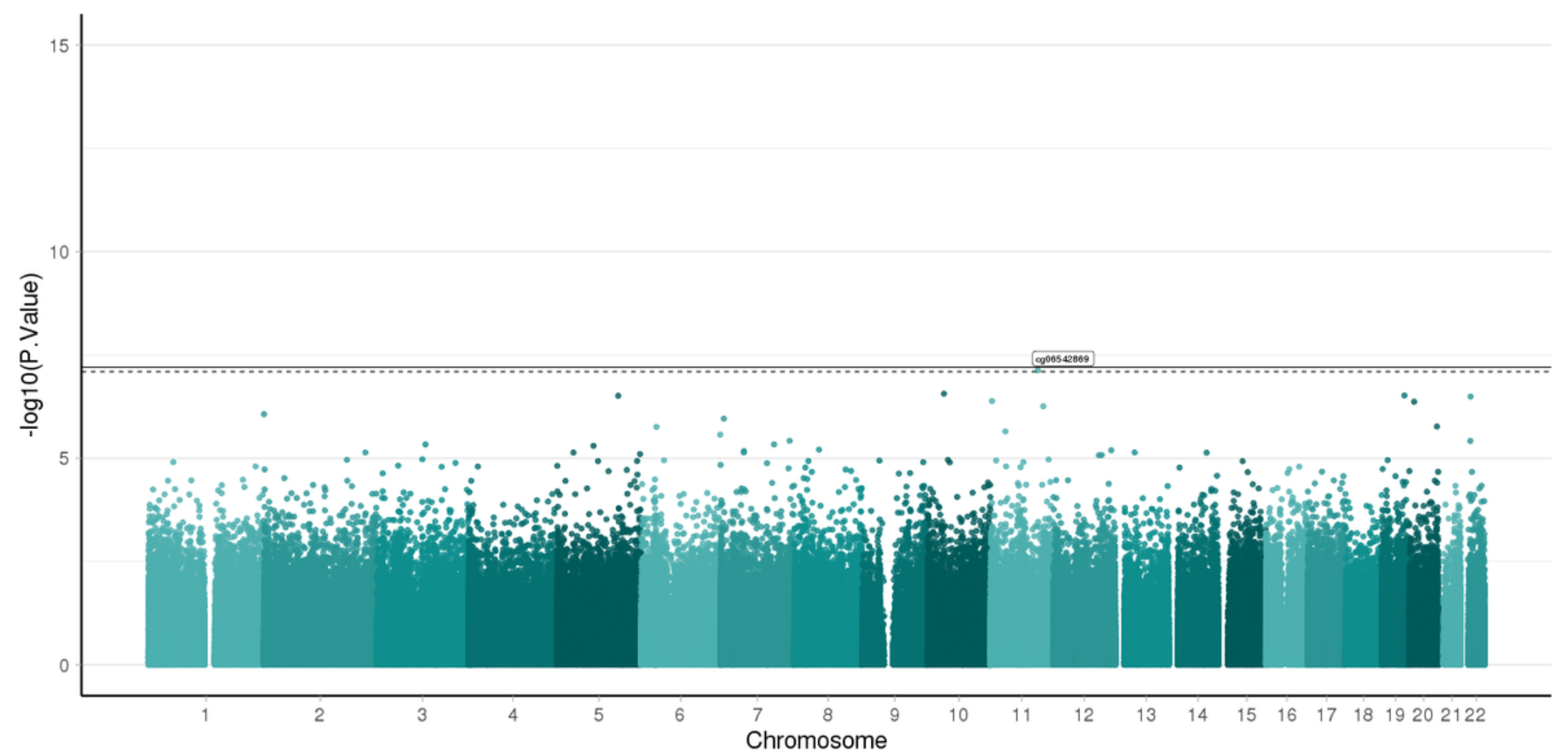

Figure 2

\section{Manhattan plot of the association between PDQ and salivary DNA methylation.}

Manhattan plots of salivary DNA methylation associated with PDQ. The x-axis represents the genomic loci of the individual $\mathrm{CpGs}$ and the $\mathrm{y}$-axis represents the -log10 (p-value). Black line: Bonferroni threshold $(p=6.183879 \mathrm{e}-08)$ and the dotted line: Multiple testing correction threshold (FDR $<0.05)$ have been added to the plot. CpGs that cross the FDR threshold are marked in the Manhattan plot. There is $1 \mathrm{CpG}$ that crosses the significance threshold. 


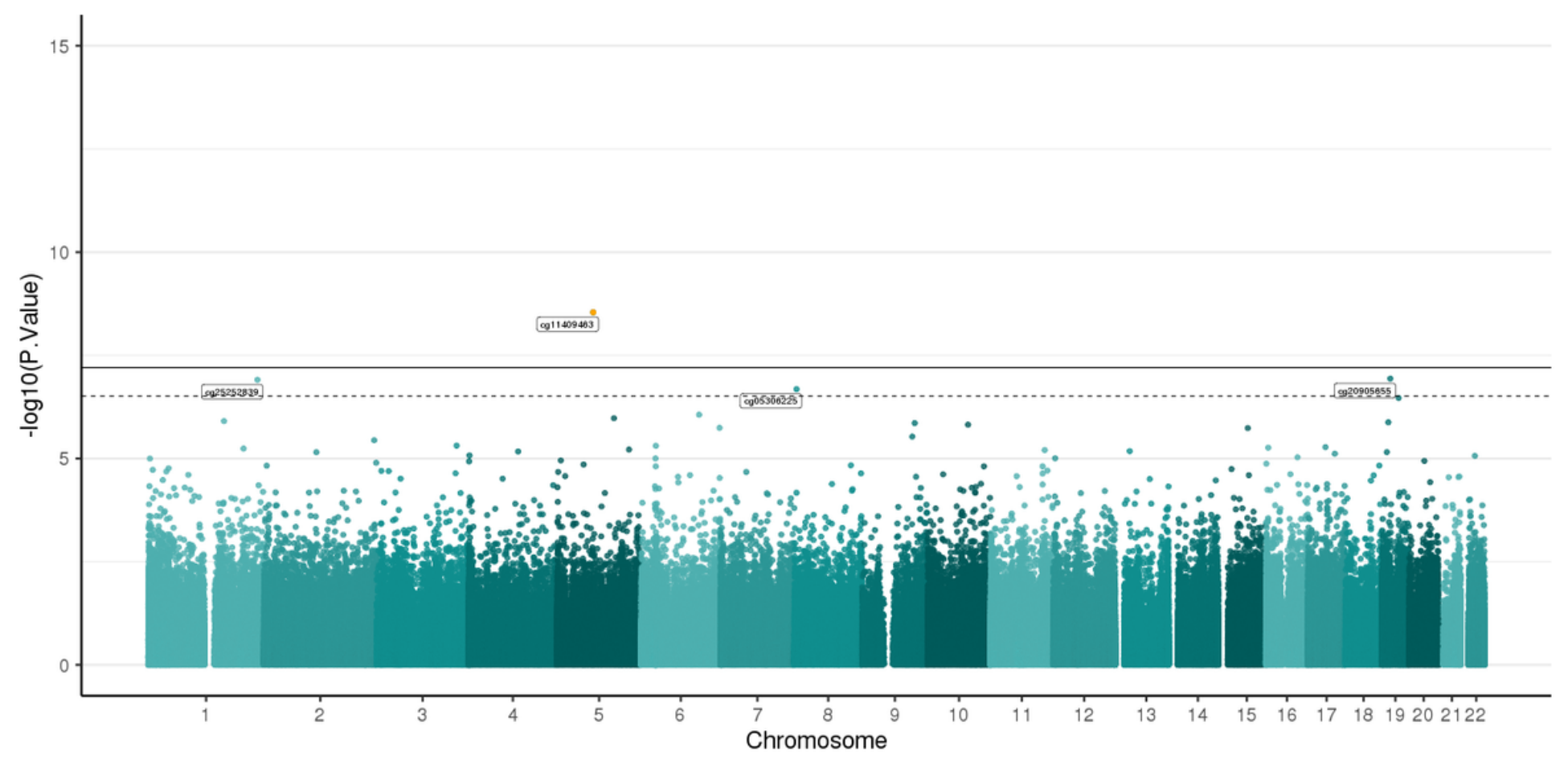

Figure 3

\section{Manhattan plot of the association between cortisol and salivary DNA methylation.}

Manhattan plots of salivary DNA methylation associated with cortisol. The x-axis represents the genomic loci of the individual $\mathrm{CpGs}$ and the $y$-axis represents the -log10 (p-value). Black line: Bonferroni threshold $(p=6.183879 \mathrm{e}-08)$ and the dotted line: Multiple testing correction threshold $(F D R<0.05)$ have been added to the plot. CpGs that cross the FDR threshold are marked in the Manhattan plot. There are four CpGs that cross the FDR multiple correction threshold. 


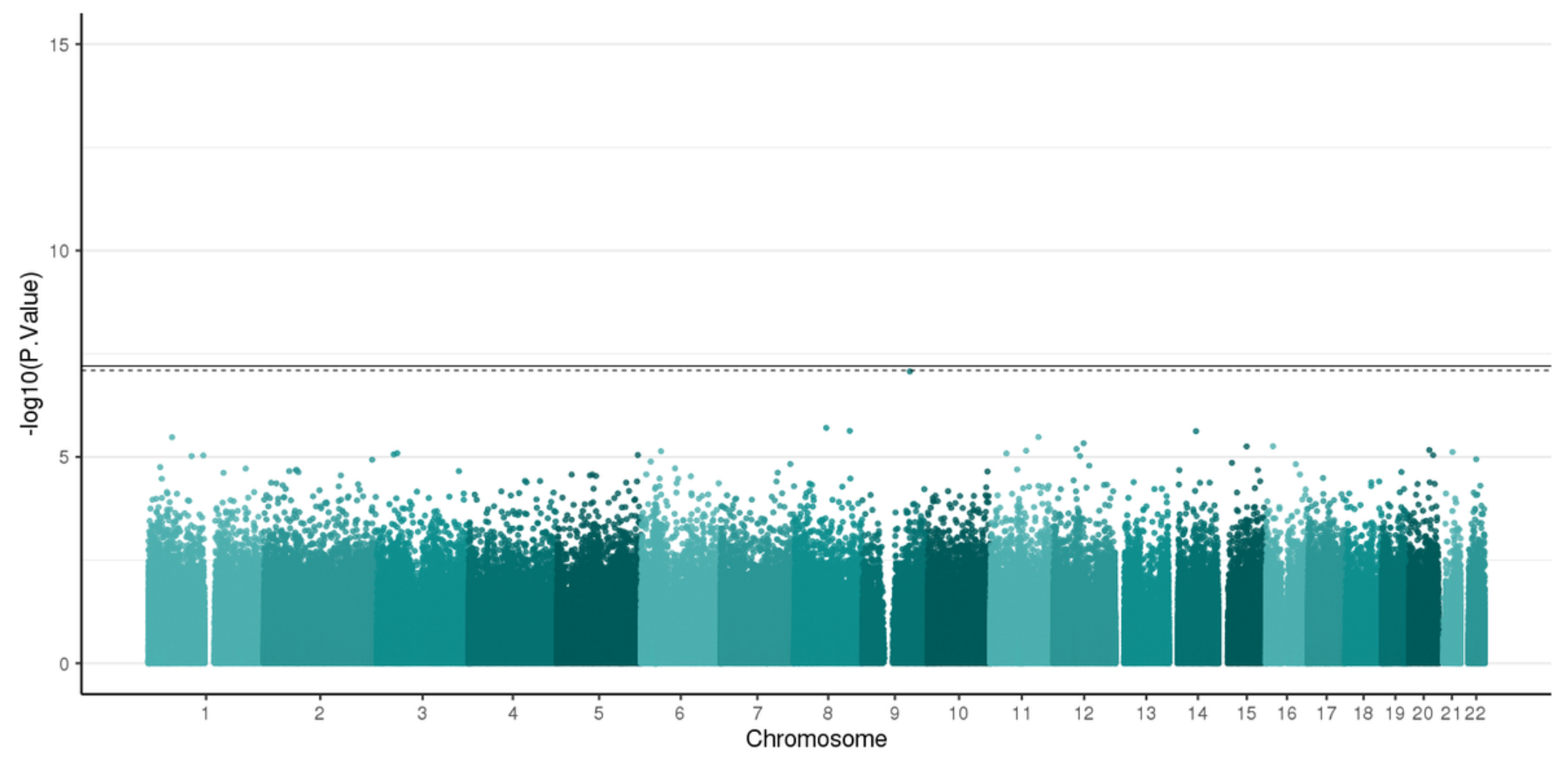

Figure 4

\section{Manhattan plot of the association between FSI and salivary DNA methylation.}

Manhattan plots of salivary DNA methylation associated with FSI (Fetal stress index). The x-axis represents the genomic loci of the individual $\mathrm{CpGs}$ and the $y$-axis represents the $-\log 10$ (p-value). Black line: Bonferroni threshold $(p=6.183879 \mathrm{e}-08)$ and the dotted line: Multiple testing correction threshold (FDR $<0.05)$ have been added to the plot. CpGs that cross the FDR threshold are marked in the Manhattan plot. There are no DMPs that cross the Bonferroni correction threshold. 


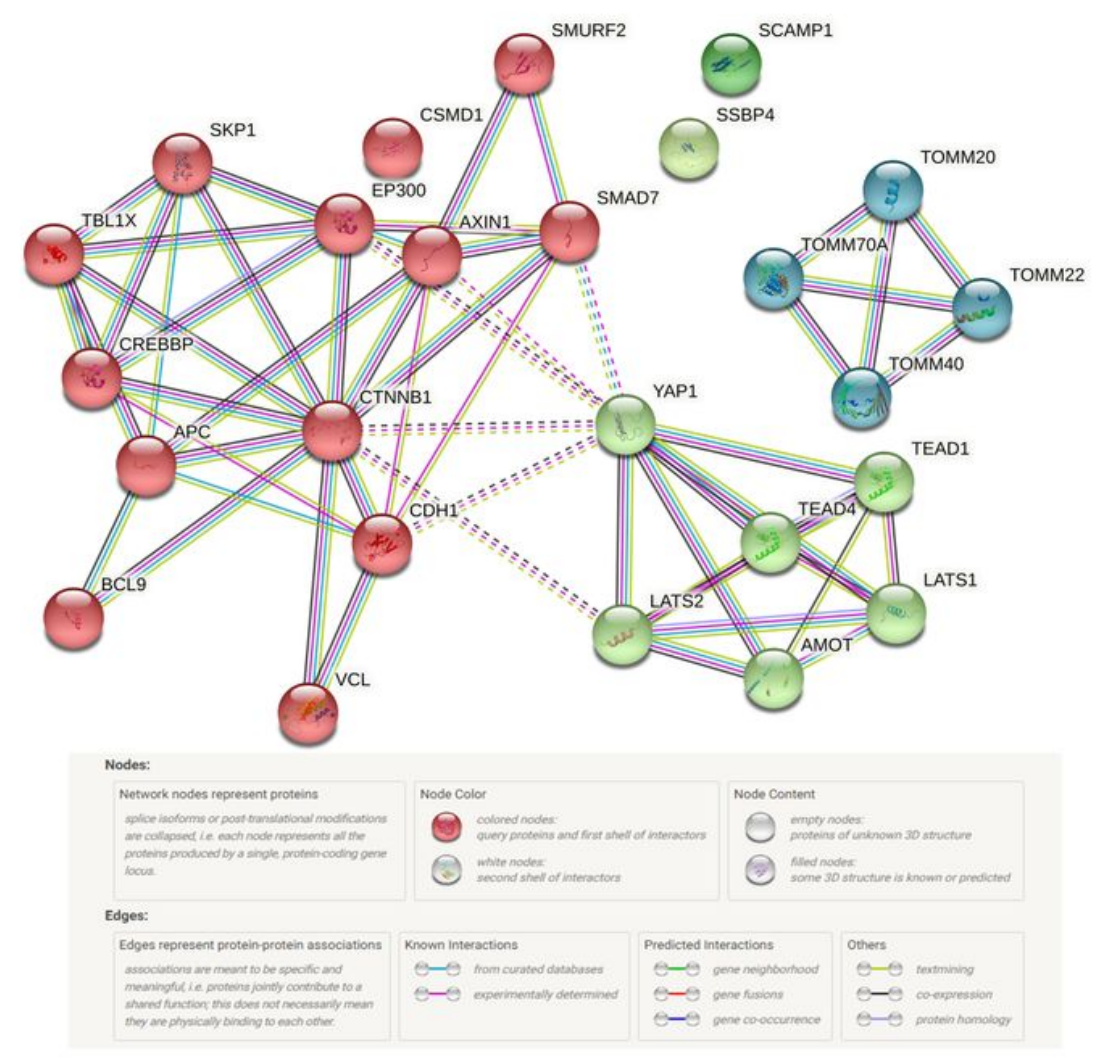

Figure 5

Network plot of significant hits from the EWAS analysis.

STRING-Db network analysis for significant hits from the association for PDQ and cortisol. Proteinprotein interaction (PPI) enrichment p-value: 3.47e-06. PPI legend by string-db.org. The permanent link is: https://version-11-5.string-db.org/cgi/network?taskld=bvfqNrZYaHe6\&sessionld=bjK7XvqNxMXe 


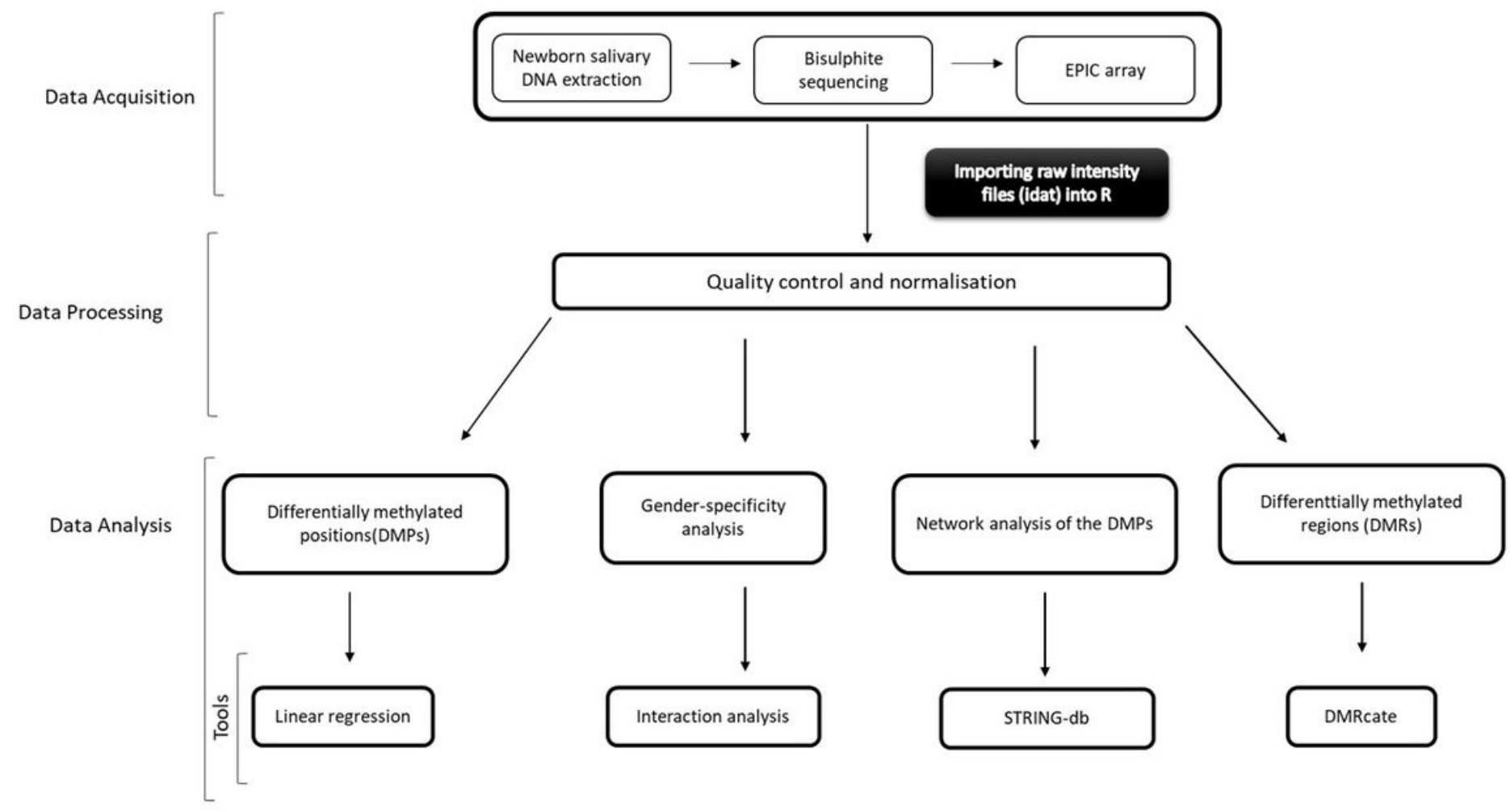

Figure 6

Overall methodological study design.

Illumina measured salivary DNA methylation using the EPIC microarray platform. The raw data were processed and quality-controlled using array-specific algorithms in R studio. Data visualization and statistical analysis identified relevant associations and derived a list of differentially methylated positions and regions.

\section{Supplementary Files}

This is a list of supplementary files associated with this preprint. Click to download.

- SupplementaryMaterial.docx 